\title{
Li Coefficients for Automorphic L-Functions
}

\author{
Jeffrey C. Lagarias \\ AT\&T Labs - Research \\ Florham Park, New Jersey 07932-0971 \\ jcl@research.att.com
}

(April 21, 2004)

\begin{abstract}
Xian-Jin Li gave a criterion for the Riemann hypothesis in terms of the positivity of the set of coefficients $\lambda_{n}=\sum_{\rho} 1-\left(1-\frac{1}{\rho}\right)^{n},(n=1,2, \ldots)$, in which $\rho$ runs over the nontrivial zeros of the Riemann zeta function. We define similar coefficients $\lambda_{n}(\pi)$ associated to principal automorphic $L$-functions $L(s, \pi)$ over $G L(N)$. We relate these cofficients to values of Weil's quadratic functional associated to the representation $\pi$ on a suitable set of test functions. The positivity of the real parts of these coefficients is a necessary and sufficient condition for the Riemann hypothesis for $L(s, \pi)$.

We derive an unconditional asymptotic formula for the coefficients $\lambda_{n}(\pi)$, in terms of the zeros of $L(s, \pi)$. Assuming the Riemann hypothesis for $L(s, \pi)$, we deduce that

$\lambda_{n}(\pi)=\frac{N}{2} n \log n+C_{1}(\pi) n+O(\sqrt{n} \log n)$, where $C_{1}(\pi)$ is a real-valued constant and the implied constant in the remainder term depends on $\pi$. We also show that there exists a entire function $F_{\pi}(z)$ of exponential type that interpolates the generalized Li coefficients at integer values. Assuming the Riemann hypothesis there is an (essentially) unique such function having exponential type at most $\pi$, and this function restricted to the real axis has a (tempered) distributional Fourier transform whose support is a countable set in $[-\pi, \pi]$ having 0 as its only limit point.
\end{abstract}

\section{Introduction}

In 1997 Xian-Jin Li 34 derived a necessary and sufficient condition for the Riemann hypothesis in terms of the positivity of the set of coefficients

$$
\lambda_{n}=\left.\frac{1}{(n-1) !} \frac{d^{n}}{d s^{n}}\left[s^{n-1} \log \xi(s)\right]\right|_{s=1} \quad n \geq 1,
$$

in which $\xi(s)=\frac{1}{2} s(s-1) \pi^{-s / 2} \Gamma\left(\frac{s}{2}\right) \zeta(s)$ is the Riemann $\xi$-function. These coefficients are expressible in terms of the zeta zeros by the formula

$$
\lambda_{n}=\sum_{\rho}^{\prime} 1-\left(1-\frac{1}{\rho}\right)^{n},
$$

in which the sum runs over the nontrivial zeros of the Riemann zeta function, counted with multiplicity, and the 'indicates that the (conditionally convergent) sum is to be interpreted as $\lim _{T \rightarrow \infty} \sum_{\{\rho:|\rho| \leq T\}}$. This summation method was termed $*$-convergence in [4]. The expression (1.2) *-converges for positive and negative integer $n$, and so defines the Li coefficients for all integers, with $\lambda_{0}=0$ and $\lambda_{-n}=\lambda_{n}$ for all $n \geq 1$. 
Li's coefficients for $n \geq 1$ appear in the power series expansion

$$
\frac{d}{d z} \log \xi\left(\frac{-z}{1-z}\right)=\frac{-1}{(1-z)^{2}} \frac{\xi^{\prime}}{\xi}\left(\frac{-z}{1-z}\right)=\sum_{n=0}^{\infty} \lambda_{n+1} z^{n} .
$$

and those for $n \leq-1$ in an an analogous formula

$$
\frac{d}{d z} \log \xi\left(\frac{1}{1-z}\right)=\frac{1}{(1-z)^{2}} \frac{\xi^{\prime}}{\xi}\left(\frac{1}{1-z}\right)=\sum_{n=0}^{\infty} \lambda_{-n-1} z^{n} .
$$

Here $\xi^{\prime}(\cdot)$ denotes the derivative with respect to $s$. Li's paper applied more generally to give a criterion for the Riemann hypothesis to hold for the (completed) Dedekind zeta functions of any algebraic number field $K$.

In [4] E. Bombieri and the author made three observations about Li's coefficients. The first is that the Li criterion can be formulated for very general sets of complex numbers $\rho$, as follows. Consider any multiset $Z$ of complex numbers $\rho$ satisfying

$$
\sum_{\rho \in Z} \frac{\Re(\rho)}{(1+|\rho|)^{2}}<\infty .
$$

If the multiset $Z$ omits the value $\rho=1$ then the sums

$$
\Re\left(\lambda_{n}(Z)\right):=\sum_{\rho} \Re\left(1-\left(1-\frac{1}{\rho}\right)^{n}\right)
$$

converge absolutely for all nonpositive integers $n \leq 0$. The positivity condition $\Re\left(\lambda_{n}(Z)\right) \geq 0$ for $n \leq 0$ then implies that all $\rho$ lie in the half-plane $\Re(s) \leq \frac{1}{2}$. There is a corresponding result for multisets that omit the value 0 : the sum (1.6) converges absolutely for $n \geq 0$, and $\Re\left(\lambda_{n}(Z)\right) \geq 0$ for $n \geq 0$ implies that all $\rho$ lie in the half-plane $\Re(s) \geq \frac{1}{2}$. On further restricting to multisets $Z$ that omit 0 and 1 and are invariant under the symmetry $\rho \mapsto 1-\bar{\rho}$, so that $\Re\left(\lambda_{n}(Z)\right)=\Re\left(\lambda_{-n}(Z)\right)$, the positivity condition $\Re\left(\lambda_{n}(Z)\right) \geq 0$ for $n>0$ implies that all $\Re(\rho)=\frac{1}{2}$. The second observation in [4. Theorem 2] was the use of the "explicit formula" of prime number theory to obtain an arithmetic expression for Li's coefficients $\lambda_{n}$ having the form

$$
\lambda_{n}=S_{\infty}(n)-S_{f}(n)+1,
$$

in which $S_{\infty}(n)$ and $S_{f}(n)$ correspond to the contributions of the archimedean place and the finite places, respectively, and the last term is a contribution from the pole at $s=0$ of $\frac{\xi(s)}{s(s-1)}$. The third observation was that each positivity condition $\lambda_{n} \geq 0$ encodes "Weil positivity" of Weil's quadratic functional for a particular test function $g_{n}(x)$.

More recently K. Maslanka [40] computed $\lambda_{n}$ for $1 \leq n \leq 2000$ and empirically studied the growth behavior of the Li coefficients. He observed that in this range they exhibited a smoothly growing dominant asymptotic term with superposed small oscillations exhibiting some internal structure. The dominant term comes from the archimedean prime contribution $S_{\infty}(n)$ in the arithmetic formula above, and the small oscillations come from the term $S_{f}(n)$ represented the finite places. Coffey [8],9] studied the "arithmetic formula" for the Li coefficients and lower bounded the archimedean prime contribution.

The object of this paper is to generalize the Li coefficients to automorphic $L$-functions and to determines some of their growth properties. The automorphic $L$-functions we treat 
are principal $L$-functions over $G L(N)$ for $\mathbb{Q}$, as given in [10, 28, 30, and [4]. These are (completed) Langlands $L$-functions attached to irreducible cuspidal unitary automorphic representations appearing in the right action of $G L\left(n, \mathbb{A}_{\mathbb{Q}}\right)$ on $L^{2}\left(G L(n, \mathbb{Q}) \backslash G L\left(n, \mathbb{A}_{\mathbb{Q}}\right)\right)$. The associated Li coefficients will be denoted $\lambda_{n}(\pi)$.

In $\S 2$ we associate to each irreducible cuspidal unitary automorphic representation $\pi$ an analogue $\xi(s, \pi)$ of the Riemann $\xi$-function. The generalized Li coefficients $\lambda_{n}(\pi)$ then correspond to $\lambda_{n}(Z)$ for the multiset $Z=Z(\pi)$ of zeros of $\xi(s, \pi)$, and we show the coefficients $\lambda_{n}(\pi)$ are well-defined as $*$-convergent series. We observe that the multiset $Z(\pi)$ is invariant under the transformation $\rho \mapsto 1-\bar{\rho}$, which implies the symmetry

$$
\lambda_{-n}(\pi)=\overline{\lambda_{n}(\pi)} .
$$

so it suffices to study $\lambda_{n}(\pi)$ for $n \geq 0$. We also observe the related symmetry

$$
\lambda_{-n}(\pi)=\lambda_{n}\left(\pi^{\vee}\right)
$$

where $\pi^{\vee}$ is the contragredient representation. The results of 4 immediately apply to give a Riemann hypothesis criterion in the form: The Riemann hypothesis holds for $L(s, \pi)$ if and only if the real parts $\Re\left(\lambda_{n}(\pi)\right)$ are nonnegative for all $n \geq 0$.

In $\S 3$ we give a "Weil positivity" interpretation of the generalized Li coefficients. We express the Weil scalar product associated to the representation $\pi$ for Li's test functions in terms of $\lambda_{n}(\pi)$. Here the imaginary parts of the $\lambda_{n}(\pi)$ appear in the scalar products. The test functions are the same for all representations $\pi$.

In $\S 4$ we give an arithmetical interpretation of the coefficients in terms of the logarithmic derivative of $\xi(s, \pi)$ expanded about at the point $s=1$. We express it as

$$
\lambda_{n}(\pi)=S_{\infty}\left(n, \pi^{\vee}\right)-S_{f}\left(n, \pi^{\vee}\right)+\delta\left(\pi^{\vee}\right),
$$

in which the two terms $S_{\infty}\left(n, \pi^{\vee}\right)$ and $S_{f}\left(n, \pi^{\vee}\right)$ reflect contributions coming from the Euler product factorization of $\xi\left(s, \pi^{\vee}\right)$ into archimedean places and finite places, respectively. The final term $\delta(\pi)=\delta\left(\pi^{\vee}\right)=1$ for the trivial representation $\pi=\pi_{\text {triv }}$ over $G L(1)$ and $\delta(\pi)=$ 0 otherwise. The contragredient representation occurs in (1.9) via the functional equation relating $\xi(s, \pi)$ and $\xi\left(1-s, \pi^{\vee}\right)$.

In $\S 5$ we obtain an unconditional asymptotic formula for the archimedean contribution $S_{\infty}(n, \pi)$. This quantity is real-valued, and we show there is a real-valued constant $C_{1}(\pi)$ such that for all $n \geq 1$,

$$
S_{\infty}(n, \pi)=\frac{N}{2} n \log n+C_{1}(\pi) n+O(1),
$$

and the implied constant in the $O(1)$ term depends on $\pi$. Here

$$
C_{1}(\pi)=\frac{N}{2}(\gamma-1-\log (2 \pi))+\frac{1}{2} \log Q(\pi),
$$

in which $\gamma$ is Euler's constant and $Q(\pi)$ is the conductor of $\pi$. In particular $C_{1}(\pi)$ does not depend on the archimedean parameters $\left\{\kappa_{j}(\pi): 1 \leq j \leq N\right\}$ of the representation $\pi$, and $C_{1}(\pi)=C_{1}\left(\pi^{\vee}\right)$.

In $\S 6$ we obtain an unconditional estimate for the finite place contribution $S_{f}(n, \pi)$ in terms of the zeros to a suitable height, by a contour integral estimate. Define the incomplete Li coefficient at height $T$ by

$$
\Omega_{n}(T, \pi)=\sum_{\substack{\rho \in Z(\pi) \\|\Im(\rho)|<T}} 1-\left(1-\frac{1}{\rho}\right)^{n}
$$


We show that for any constant $\beta>\frac{1}{\log 2}$ there holds

$$
S_{f}(n, \pi)=\Omega_{n}\left(\sqrt{\beta n}, \pi^{\vee}\right)+O(\sqrt{n}),
$$

where $\pi^{\vee}$ is the contragredient representation, and the implied constant in the $O$-notation depends on both $\beta$ and $\pi$. If the Riemann hypothesis holds for $L(s, \pi)$, hence for $L\left(s, \pi^{\vee}\right)$ by the functional equation, then we have

$$
S_{f}(n, \pi)=O(\sqrt{n} \log n) .
$$

Furthermore if the Riemann hypothesis holds up to height $T$, then a bound of shape (1.14) holds for all $n \leq \frac{T^{2}}{4(\log T)^{2}}$, with the implied O-constant depending on $\pi$.

These results are summarized in the following theorem.

Theorem 1.1. Let $\pi$ be an irreducible cuspidal unitary automorphic representation for $G L(N)$ over $\mathbb{Q}$. There for $n \geq 0$ and any fixed $\beta$ with $\beta>\frac{1}{\log 2}$ there holds

$$
\lambda_{n}(\pi)=\frac{N}{2} n \log n+C_{1}(\pi) n-\Omega_{n}(\sqrt{\beta n}, \pi)+O(\sqrt{n}),
$$

in which $C_{1}(\pi)$ is real-valued and the implied constant in the $O$-notation depends on $\beta$ and $\pi$. If the Riemann hypothesis is valid for $L(s, \pi)$ then for all $n \geq 1$,

$$
\Omega_{n}\left(\sqrt{\beta n}, \pi^{\vee}\right)=O(\sqrt{n} \log n) .
$$

where the implied constant in the $O$-notation depends on $\pi$.

Theorem 1.1 follows on combining Lemma 4.2, Theorem 5.1 and Theorem 6.1, and the fact $C_{1}(\pi)=C_{1}\left(\pi^{\vee}\right)$.

If the Riemann hypothesis for $L(s, \pi)$ is false then $\lambda_{n}(\pi)$ will sometimes be very large, of size exponential in $n$, coming from the term $\Omega_{n}(\sqrt{\beta n}, \pi)$. This was observed for the Riemann zeta function in [4, Theorem 1(c)].

In $\S 7$ we construct for each $\pi$ an entire function $F_{\pi}(z)$ that interpolates the Li coefficients at integer values, i.e. $F_{\pi}(n)=\lambda_{n}(\pi)$ for all $n \in \mathbb{Z}$, and which is of order one and exponential type. Assuming the Riemann hypothesis for $L(s, \pi)$, this function $F_{\pi}(z)$ can be chosen to have exponential type at most $\pi$. It is then almost uniquely ${ }^{1}$ characterized by the interpolation property, and has a Fourier transform well-defined as a tempered distribution, whose support is a countable closed set in $[-\pi, \pi]$ having 0 as its only limit point. This interpolation function $F_{\pi}(z)$ appears to be a new object associated to the zeta zeros. We do not know any relation of it to various other functions constructed from the zeta zeros such as Cramer's $V$-function (11], 23, [19, 33]) or functions studied by Voros [48, 49].

The results of this paper explain some of the empirical observations of K. Maslanka [40]. The asymptotic formula (1.14) applied to $\pi_{t r i v}$, explains the observed behavior of the small Li coefficients. Since the non-trivial zeros of $\zeta(s)$ are known to lie on the critical line up to height $T \approx 10^{9}$ we may expect the first $10^{16} \mathrm{Li}$ coefficients will also exhibit similar asymptotic behavior, i.e. the term $\left|S_{f}(n)\right|$ will remain small over this range. Our results only establish the "expected" square-root cancellation. Maslanka's computations of $S_{f}(n)$ allow the possibility that the term $\left|S_{f}(n)\right|$ is of smaller order of growth. If so it might represent extra correlations

\footnotetext{
${ }^{1}$ Zeros at the central critical value $s=\frac{1}{2}$ require special treatment, see Theorem 7.1
} 
among the zeta zeros, which would be extremely interesting if true. On the other hand, it may be that the true order of growth of the error term is not visible in the first few thousand coefficients. E. Bombieri has observed an analogy with the differences $|\pi(x)-\operatorname{Li}(x)|$. Here it is proved that $|\pi(x)-\operatorname{Li}(x)|>x^{\frac{1}{2}}$ infinitely often, but we have $|\pi(x)-\operatorname{Li}(x)|<15$ for $x<2000$. The approximate formula (1.15) gives no information on the precise spectral nature of the "small oscillations" in $S_{f}(n)$. Perhaps further information can be extracted from the Fourier transform of the interpolating function defined in $\S 7$.

While this paper was being completed, an asymptotic formula for the Li coefficients $\lambda_{n}$ was announced by A. Voros [50], under the Riemann hypothesis. Comparison of his formula with that obtained here in (5.30) led to a simplification of the expression for $\left.C_{(} \pi\right)$ in Theorem [5.1,

Acknowlegments. The author thanks E. Bombieri, Xian-Jin Li and A. M. Odlyzko for helpful comments, K. Maslanka for communicating various numerical constants and graphs of Li coefficients, and M. Coffey for communicating preliminary versions of his work.

\section{Li Coefficients for Automorphic $L$-functions}

We recall basic facts about principal $L$-functions $L(s, \pi)$ attached to irreducible cuspidal automorphic representations of $G L(N)$, as in Jacquet 30] and Rudnick and Sarnak [Sect. 2] 44, see also Gelbart and Miller [21, Sect. 7.2]. These $L$-functions are associated to $G L(n, \mathbb{Q}) \backslash G L\left(n, \mathbb{A}_{\mathbb{Q}}\right)$, and they are more precisely denoted $L(s, \pi, \rho)$ in which the Langlands $L$-group ${ }^{L} G=G L(N, \mathbb{C})$ and $\rho:{ }^{L} G \rightarrow G L(N, \mathbb{C})$ is the standard representation.

For the trivial representation $\pi_{\text {triv }}$ of $G L(1)$ we have the completed automorphic $L$-function $\Lambda\left(s, \pi_{\text {triv }}\right)=\pi^{-\frac{s}{2}} \Gamma\left(\frac{s}{2}\right) \zeta(s)$. This function has simple poles at $s=0$ and $s=1$. Aside from this representation, all other $\Lambda(s, \pi)$ are entire functions.

Each completed automorphic $L$-function $\Lambda(s, \pi)$ has an Euler product factorization

$$
\Lambda(s, \pi):=Q(\pi)^{\frac{s}{2}} L_{\infty}(s, \pi) L(s, \pi) .
$$

Here $Q(\pi)$ is a positive integer called the conductor of the representation $\pi$, and the archimedean factor is

$$
L_{\infty}(s, \pi)=\prod_{j=1}^{N} \Gamma_{\mathbb{R}}\left(s+\kappa_{j}(\pi)\right),
$$

in which $\kappa_{j}(\pi)$ are certain constants and

$$
\Gamma_{\mathbb{R}}(s):=\pi^{-\frac{s}{2}} \Gamma\left(\frac{s}{2}\right) .
$$

The (finite) $L$-function $L(s, \pi)$ is given by an Euler product over the finite places

$$
\begin{aligned}
L(s, \pi) & =\prod_{p} \prod_{j=1}^{N}\left(1-\alpha_{p, j}(\pi) p^{-s}\right)^{-1} . \\
& =\sum_{n=1}^{\infty} a_{n}(\pi) n^{-s} .
\end{aligned}
$$

This Euler product and its associated Dirichlet series converges absolutely in a half-plane specified below. The functions $\Lambda(s, \pi)$ satisfy a functional equation

$$
\Lambda(s, \pi)=\epsilon(\pi) \Lambda\left(1-s, \pi^{\vee}\right),
$$


in which $\epsilon(\pi)$ is a constant of absolute value one, and $\pi^{\vee}$ denotes the contragredient representation. The contragredient representation has $L$-function

$$
L\left(s, \pi^{\vee}\right)=\sum_{n=1}^{\infty} \overline{a_{n}(\pi)} n^{-s}
$$

and archimedean factor

$$
L_{\infty}\left(s, \pi^{\vee}\right)=L_{\infty}(s, \pi),
$$

and has conductor $Q\left(\pi^{\vee}\right)=Q(\pi)$. The functional equation implies that $\epsilon\left(\pi^{\vee}\right)=\overline{\epsilon(\pi)}$. The functions $\Lambda(s, \pi)$ are bounded in vertical strips, with exponential decay as $|\Im(s)| \rightarrow \infty$, with $-B<\Re(s)<B$ for any fixed $B$.

We define the $\xi$-function $\xi(s, \pi)$ associated to $\pi$ by

$$
\xi(s, \pi):=s^{-e(0, \pi)}(s-1)^{-e(1, \pi)}\left(\frac{1}{\sqrt{(-1)^{e\left(\frac{1}{2}\right)} \epsilon(\pi)}} \Lambda(s, \pi)\right),
$$

where $e\left(s_{0}, \pi\right)$ denotes the order of a zero or pole of $\Lambda(s, \pi)$ at $s=s_{0}$, with a simple pole of order -1 . We have $e(0, \pi)=e(1, \pi)$ by the functional equation, and this definition ensures that $\xi(s, \pi)$ is holomorphic and nonzero at $s=0$ and 1 . In this definition the square roots must be chosen consistently so that

$$
\sqrt{(-1)^{e\left(\frac{1}{2}\right)} \epsilon(\pi)} \cdot \sqrt{(-1)^{e\left(\frac{1}{2}\right)} \epsilon\left(\pi^{\vee}\right)}=1 .
$$

There remains a choice of sign, which can be removed by the requirement that $\xi\left(\frac{1}{2}+i t, \pi\right)>0$ hold for small positive $t$, as justified in the result below. For the trivial representation $\pi_{\text {triv }}$ on $G L(1)$ we have $e\left(0, \pi_{\text {triv }}\right)=e\left(1, \pi_{\text {triv }}\right)=-1$, and $\xi\left(s, \pi_{\text {triv }}\right)=2 \xi(s)$. This convention is forced if we wish to have entire functions in all cases, for we must remove the poles at $s=0$ and $s=1$ for the case $\pi_{t r i v}$.

The following theorem collects together analytic facts about automorphic $L$ - functions.

Theorem 2.1. $\quad$ Let $\pi$ be a irreducible cuspidal unitary automorphic representation of $G L(n)$ over $\mathbb{Q}$.

(1) The ordinary Dirichlet series $L(s, \pi)=\sum_{n=1}^{\infty} a_{n}(\pi) n^{-s}$ converges absolutely in the half-plane $\Re(s)>1$. We have for all $n \geq 1$ that

$$
\left|a_{n}(\pi)\right| \leq C(\pi) d(n) n^{\frac{N}{2}}
$$

for some $C(\pi)>0$ depending on $\pi$, and $d(n)$ is the number of divisors of $n$.

(2) The archimedean factors $\Gamma_{\mathbb{R}}\left(s+\kappa_{j}(\pi)\right)$ in the Euler product $\Lambda(s, \pi)$ satisfy

$$
\Re\left(\kappa_{j}(\pi)\right)>-\frac{1}{2} .
$$

The quantities $\left\{\kappa_{j}(\pi): 1 \leq j \leq N\right\}$ are permuted under complex conjugation, so that

$$
L_{\infty}(s, \pi)=\overline{L_{\infty}(\bar{s}, \pi)} .
$$

(3) The zeros of $\Lambda(s, \pi)$ all lie in the open critical strip $0<\Re(s)<1$. In particular $\Lambda(s, \pi)$ is non-vanishing on the lines $\Re(s)=0$ and $\Re(s)=1$. 
(4) The counting function $N_{\pi}^{+}(T)$ for zeros of $\Lambda(s, \pi)$ with $0 \leq \Im\left(\rho_{\pi}\right)<T$ and $N_{\pi}^{-}(T)$ for $-T \leq \Im\left(\rho_{\pi}\right) \leq 0$ each satisfy

$$
N_{\pi}^{ \pm}(T)=\frac{N}{2 \pi} T \log T+\frac{1}{2} C_{0}(\pi) T+O(\log T)
$$

as $T \rightarrow \infty$. Here

$$
C_{0}(\pi)=\frac{1}{\pi} \log Q(\pi)-\frac{N}{\pi}(1+\log 2),
$$

in which $Q(\pi)$ is the conductor of $\pi$, and the O-constant depends on $\pi$.

(5) $\xi(s, \pi)$ satisfies the functional equation

$$
\xi(s, \pi)=(-1)^{k} \xi\left(1-s, \pi^{\vee}\right),
$$

where $k=e\left(\frac{1}{2}, \pi\right)=e\left(\frac{1}{2}, \pi^{\vee}\right)$ is the order of the zero of $\xi(s, \pi)$ at $s=\frac{1}{2}$. It is real-valued on the critical line $\Re(s)=\frac{1}{2}$, so the multiset $Z(\pi)$ is invariant under the map $\rho \mapsto 1-\bar{\rho}$.

(6) The function $\xi(s, \pi)$ is an entire function of order one and maximal type. It is bounded in vertical strips $-B<\Re(s)<B$ for any finite $B$, and has rapid decrease there as $|\Im(s)| \rightarrow \infty$.

Proof. (1) Those irreducible cuspidal automorphic representations $\pi$ that arise as subrepresentations of the right regular representation on $L^{2}\left(G L(n, \mathbb{Q}) \backslash G L\left(n, \mathbb{A}_{\mathbb{Q}}\right), d \mu_{\text {Haar }}\right)$ are necessarily unitary. We use the bound of Jacquet and Shalika 31] 31, Theorem 5.3], that RankinSelberg convolutions $L\left(s, \pi_{1} \times \pi_{2}\right)$ of unitary irreducible cuspidal automorphic representations $\pi_{1}, \pi_{2}$ have Dirichlet series that converge in $\Re(s)>1$. We take $\pi_{1}=\pi$ and $\pi_{2}$ to be the trivial representation. (See also [28, Sect. 5.10].)

Jacquet and Shalika [31, Corollary 2.5] also derive the bound that all unramified primes in the Euler product have $\left|\alpha_{j, p}\right|<\sqrt{p}$. All but finitely many primes are unramified, and therefore multiplying out the Euler product yields the bound (2.8). Better bounds are known for $\left|\alpha_{j, p}\right|$ which give larger regions of absolute convergence. Rudnick and Sarnak [44, Prop. 5.1] show for irreducible cuspidal automorphic repesentations $\pi$ of $G L(N)$ unramified at $p$ satisfy

$$
\left|\alpha_{j, p}(\pi)\right| \leq p^{\frac{1}{2}-\frac{1}{N^{2}+1}}
$$

The generalized Ramanujan conjecture formulated in [29] asserts that each $\left|\alpha_{j, p}\right|=1$ at unramified places $p$. It is known to be true for principal $L$-functions over $G L(1)$ (Dirichlet $L$-functions).

(2) The inequality $\Re(s)>-\frac{1}{2}$ is established in Rudnick and Sarnak 44, eqn. (2.5) and Sect. 5.3]. There exist ramified archimedean representations over $G L(2)$ for which some $\Re\left(\kappa_{j}(\pi)\right.$ is arbitrarily large. However Luo, Rudnick and Sarnak [39] show that unramified archimedean representations ${ }^{2}$ satisfy the stronger bound

$$
|\Re(\kappa(\pi))| \leq \frac{1}{2}-\frac{1}{N^{2}+1} .
$$

The Ramanujan conjecture at the archimedean places asserts for an unramified representation that all $\Re\left(\kappa_{j}(\pi)\right)=0$. Its truth would imply that the bound (2.9) could be improved to $\Re\left(\kappa_{j}(\pi)\right) \geq 0$ in the general case.

\footnotetext{
${ }^{2} \mathrm{~A}$ representation is unramified if it is identically one on the maximal compact subgroup of all the archimedean components of $G L(N)$. A Dirichlet character $\chi$ for $G L(1)$ with $\chi(-1)=-1$ corresponds to an automorphic representation for $G L(1)$ ramified at the archimedean place, with $\kappa(\chi)=1$.
} 
The symmetry under complex conjugation of the $\kappa_{j}(\pi)$ holds because the local factors in the Euler product are equivalent to contragredient (see 20, Theorem 2] and 44, Sect. 2.2]), which gives (2.10).

(3) Iwaniec and Kowalski 28, Theorem 5.42] show non-vanishing of cuspidal automorphic $L$-functions for $G L(N)$ on the line $\Re(s)=1$, and also obtain a zero-free region inside the critical strip. The nonvanishing on $\Re(s)=0$ comes from the functional equation (2.4) for $\Lambda(s, \pi)$.

(4) The asymptotic formula for $N_{\pi}(T)$ is essentially determined by the archimedean factors in the Euler product for $\Lambda(s, \pi)$. The counting result the zero density from $-T$ to $T$ appears as [28, Theorem 5.8], in which $q=q(\pi)$ is the conductor of $\pi$ (see [28, Sec. 5.1]). The error term given is $O(\log \mathfrak{q}(\pi, i T))$, with an absolute constant, which involves the analytic conductor

$$
\mathfrak{q}(\pi, s):=Q(\pi) \prod_{j=1}^{N}\left(\left|s+\kappa_{j}(\pi)\right|+3\right) .
$$

Since we regard $\pi$ as fixed, this yields $O(\log T)$, with the $O$-constant depending on $\pi$. To get the bound (4) on the upper and lower critical strip, the contour integral proof in 28 must be modified to split into two contours with a cut along the real axis, which goes off it in small circles to avoid poles at the trivial zeros. (We omit the details.)

(5) The definition of $\xi(s)$ ensures that the functional equation (2.8) holds up to a sign, which depends on the multiplicity $(\bmod 2)$ of a zero of $\Lambda(s, \pi)$ at $s=\frac{1}{2}$. Now the functions $\Lambda(s, \pi)$ have the symmetry

$$
\Lambda(s, \pi)=\overline{\Lambda\left(\bar{s}, \pi^{\vee}\right)} .
$$

which follows from (2.10) and (2.5). In consequence (2.7) gives

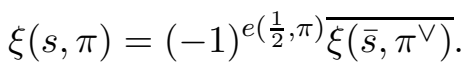

We deduce that

$$
\xi\left(\frac{1}{2}+i t, \pi\right)=(-1)^{e\left(\frac{1}{2}, \pi\right)} \xi\left(\frac{1}{2}-i t, \pi^{\vee}\right)=\overline{\xi\left(\frac{1}{2}+i t, \pi\right)} .
$$

Thus $\xi(s, \pi)$ is real on the critical line. The invariance of the multiset $Z(\pi)$ under $\rho \mapsto 1-\bar{\rho}$ now follows from the reflection principle.

(6) Godemont and Jacquet [22, Theorem 13.8] showed for cuspidal automorphic representations $\pi$ that $\Lambda(s, \pi)$ is meromorphic and bounded in vertical strips, with finitely many poles, and that the ordinary Dirichlet series $L(s, \pi)$ has a nonempty half-plane of absolute convergence, (See also Jacquet [30, Theorem 6.2]). The assumption that $\pi$ is irreducible and cuspical gives that they are entire functions, aside from the trivial representation $\pi_{t r i v}\|\cdot\|^{s}$ of $G L(1)$.

In the vertical strip $-B \leq \Im(s) \leq B$ it satisfies

$$
|\xi(s, \pi)| \leq C(\pi, B) e^{-N \frac{\pi}{2}|\Im(s)|}
$$

as $|\Im(s)| \rightarrow \infty$. The exponential decay on vertical lines holds for $\Re(s) \geq 1+\epsilon$, coming from exponential decay of the archimedean factor and $|L(s, \pi)|=O(1)$. It then holds for $\Re(s) \leq-\epsilon$ by the functional equation, and then in between by the Phragmen-Lindelof principle.

In the half-plane $\Re(s) \geq 1+\epsilon$ we have $|\Lambda(s, \pi)|=O(1)$ because its Dirichlet series representation converges absolutely. It follows that on this half-plane $|\Lambda(s, \pi)|=O\left(e^{N|s|(\log |s|+1)}\right)$, with the growth rate coming from the archimedean factors, which can be bounded by Stirling's formula. The functional equation now shows that the same bound holds on the half plane 
$\Re(s) \leq-\epsilon$. Since $|\Lambda(s, \pi)|$ is known to be bounded on the vertical strip $-\epsilon \leq \Re(s) \leq 1+\epsilon$, it follows that $|\Lambda(s)|=O\left(e^{N|s|(\log (|s|+2)}\right)$ for all $s$. Thus $\Lambda(s)$ is an entire function of order one. It is of maximal type because its growth-rate is faster than $e^{R|s|}$ for any finite $R$ along the positive real axis, coming from the archimedean factors. The function $|\xi(s, \pi)|$ inherits these properties, since it differs by at most a polynomial factor from $\Lambda(s, \pi)$.

We let $Z(\pi)$ denote the multi-set of zeros of $\xi(s, \pi)$ (counted with multiplicity) which is the same as that of $\Lambda(s, \pi)$ except possibly at $s=0$ and $s=1$.

Lemma 2.1. For any principal L-function $L(s, \pi)$ for $G L(N)$ the power sums

$$
\sigma_{n}(\pi):=\sum_{\rho \in Z(\pi)} \frac{1}{\rho^{n}}, \quad n \geq 1
$$

are absolutely convergent for $n \geq 2$, and are $*$-convergent for $n=1$. The real parts of these sums are absolutely convergent for all $n \geq 1$.

Proof. The absolute convergence for $n \geq 2$ follows easily from the zero-counting bound (2.11).

The $*$-convergence for $\sigma_{1}(\pi)$ follows from the asymptotics (2.11) for the zeros. Here the zeros below and above the real axis are paired in increasing order of their imaginary parts (in absolute values) and using the asymptotic formulas in Theorem 2.1(4) we get $*$-convergence by partial summation. It is important that the remainder term in (2.11) be $O\left(T^{1-\delta}\right)$ for some $\delta>0$. (In general the zeros are not symmetric about the real axis. The symmetry $\rho \mapsto 1-\bar{\rho}$ is of no help in proving $*$-convergence.) Finally, for any zero $\rho=\beta+i \gamma$ we have

$$
\Re\left(\frac{1}{\rho}\right)=\frac{\beta}{\beta^{2}+\gamma^{2}}=O\left(\frac{1}{\gamma^{2}}\right),
$$

which gives absolute convergence for $n=1$ of the real parts of the terms in the sum (2.16).

Lemma 2.2. For all irreducible cuspidal unitary automorphic representations on $G L(N)$ the sums

$$
\lambda_{n}(\pi):=\sum_{\rho \in Z(\pi)} 1-\left(1-\frac{1}{\rho}\right)^{n}
$$

are $*$-convergent for all $n \in \mathbb{Z}$. They are given by

$$
\lambda_{n}(\pi)=\sum_{j=1}^{n}(-1)^{j-1}\left(\begin{array}{l}
n \\
j
\end{array}\right) \sigma_{j}(\pi)
$$

with $\lambda_{0}(\pi)=0$. They satisfy

$$
\lambda_{-n}(\pi)=\overline{\lambda_{n}(\pi)}=\lambda_{n}\left(\pi^{\vee}\right)
$$

Proof. We have

$$
\sum_{|\rho|<T} 1-\left(1-\frac{1}{\rho}\right)^{n}=\sum_{|\rho|<T} \frac{n}{\rho}+\sum_{j=2}^{n}(-1)^{j-1}\left(\begin{array}{c}
n \\
j
\end{array}\right)\left(\sum_{|\rho|<T} \frac{1}{\rho^{j}}\right) .
$$


On letting $T \rightarrow \infty$, Lemma 2.1 shows that the first sum on the right $*$-converges and the second sum on the right converges absolutely. This gives (2.18).

The symmetry $\lambda_{-n}(\pi)=\overline{\lambda_{n}(\pi)}$ is inherited from the symmetry that if $\rho=\beta+i \gamma$ is a zero of $\xi(s)$, then so is $1-\bar{\rho}=1-\beta+i \gamma$, with the same multiplicity. This holds by the reflection principle since $\xi(s)$ is real on the critical line. That is, the multiset of zeros is invariant under the map $\rho \mapsto 1-\bar{\rho}$. Now we have

$$
1-\left(1-\frac{1}{\rho}\right)^{-n}=1-\left(\frac{\rho-1}{\rho}\right)^{-n}=1-\left(\frac{-\rho}{1-\rho}\right)^{n}=1-\overline{\left(1-\left(\frac{1}{1-\bar{\rho}}\right)^{n}\right.} .
$$

and this gives the result.

We can now state a general version of Li's criterion.

Theorem 2.2. Let $\pi$ be an irreducible cuspidal unitary automorphic representation of $G L(n)$. The following conditions are each equivalent to the Riemann hypothesis for $\xi(s, \pi)$.

(1) For all $n \geq 1$,

$$
\Re\left(\lambda_{n}(\pi)\right) \geq 0 .
$$

(2) For each $\epsilon>0$, there is a positive constant $C(\epsilon)$ such that

$$
\Re\left(\lambda_{n}(\pi)\right) \geq-C(\epsilon) e^{\epsilon n} \quad \text { for all } \quad n \geq 1 .
$$

(3) The generalized Li coefficients $\lambda_{n}(\pi)$ satisfy

$$
\lim _{n \rightarrow \infty}\left|\lambda_{n}(\pi)\right|^{\frac{1}{n}} \leq 1
$$

Proof. Theorem 2.1 gives that the multiset $Z(\pi)$ omits the values 0 and 1 and is invariant under the symmetry $\rho \mapsto 1-\bar{\rho}$. The equivalence of (1) and (2) to the Riemann hypothesis for $Z(\pi)$ follows from the Corollary in Theorem 1 of [4].

We now show the equivalence of (3) to the Riemann hypothesis. The $\lambda_{n}(\pi)$ are identifiable as the power series coefficients around $z=0$ of

$$
\frac{d}{d z} \log \xi\left(\frac{-z}{1-z}, \pi\right)=\frac{-1}{(1-z)^{2}} \frac{\xi^{\prime}}{\xi}\left(\frac{-z}{1-z}, \pi\right)=\sum_{n=0}^{\infty} \lambda_{n+1}(\pi) z^{n} .
$$

The map $z \mapsto s=-\frac{z}{1-z}$ conformally maps the unit disk to the half-plane $\Re(s) \geq \frac{1}{2}$, and the point $z=0$ corresponds to $s=1$, a point where $\frac{\xi^{\prime}}{\xi}(s)$ is holomorphic. Assuming the Riemann hypothesis holds for $\xi(s, \pi)$, the function in (2.23) is holomorphic in the unit disk, hence its power series coefficients satisfy (2.22). Conversely, if (2.22) holds, then the function (2.23) is holomorphic in the unit disk, hence $\frac{\xi^{\prime}}{\xi}(s, \pi)$ has no singularity in $\Re(s)>\frac{1}{2}$. The functional equation then shows it has no singularity in $\Re(s)<\frac{1}{2}$ so the Riemann hypothesis holds for $\xi(s, \pi)$.

\section{Li Coefficients and Weil's Quadratic Functional}

A. Weil [51] formulated the "explicit formula" of prime number theory in terms of distributions, and using the Fourier transform on the real line. He gave a necessary and sufficient condition for the Riemann hypothesis in terms of the positivity of a quadratic functional on a suitable space of test functions on the real line. There is a natural generalization of Weil's 
quadratic functional associated to the completed $L$-function $\Lambda(s, \pi)$ of any irreducible cuspidal automorphic representation $\pi$ of $G L(N)$, which we shall conisder here.

We first note that, using an exponential change of variable, the "explicit formula" is expressible in terms of test functions on the positive real line $\mathbb{R}_{>0}$, with the Fourier transform replaced by the Mellin transform. This is the framework for the "explicit formula" taken in 3], 4. One can now make a second change of variable, using the Mellin transform itself, to transfer Weil's functional to a quadratic functional on a space of test functions contained inside $L^{2}\left(\frac{1}{2}+i \mathbb{R}, \frac{d t}{2 \pi}\right)$; these test functions are the Mellin transforms of the test functions on $\mathbb{R}_{>0}$ above. This is the viewpoint we use in this paper; it was taken in Burnol [5]. The resulting test functions necessarily have the property of being analytic in some open domain that includes the critical line $\Re(s)=\frac{1}{2}$ in its interior. If the Riemann hypothesis is not assumed, this set of test functions must be restricted to functions analytic in a region containing the closed critical strip.

We consider as test functions the vector space $\mathcal{A}$ of all functions $F(s)$ holomorphic in the strip $0<\Re(s)<1$ which satisfy a uniform bound $F(s)=O\left(\frac{1}{|s|}\right)$ in the strip outside ${ }^{3}$ the region $|\Im(s)| \leq 1$, with $O$-constant depending on the function. The class $\mathcal{A}$ is closed under the action of the involution $\tilde{G}(s):=G(1-s)$. These functions are completely determined by their values on the critical line $\Re(s)=\frac{1}{2}$ by analytic continuation, and the bound on their growth ensures that they belong to $L^{2}\left(\frac{1}{2}+i \mathbb{R}, \frac{d t}{2 \pi}\right)$. Under the inverse Mellin transform they convert to a class of smooth test functions contained in $L^{2}\left(\mathbb{R}_{>0}, \frac{d x}{x}\right)$ of the type considered in [4].

Given $F(s), G(s) \in \mathcal{A}$ we define the Weil scalar product associated to the automorphic representation $\pi$ by

$$
\langle F, G\rangle_{\mathcal{W}(\pi)}:=\sum_{\rho \in Z(\pi)} F(\rho) \overline{G(1-\bar{\rho})} .
$$

The sum on the right counts zeros with multiplicity, and it converges absolutely due to the growth bound on $F$ and $G$ for large $|s|$. This scalar product is linear in the first factor and conjugate-linear in the second factor. The multiset of automorphic $L$-function zeros $Z(\pi)$ (counting multiplicities) is invariant under the involution $\rho \rightarrow 1-\bar{\rho}$. This yields the Hermitian symmetry

$$
\langle F, G\rangle_{\mathcal{W}(\pi)}=\overline{\langle G, F\rangle}_{\mathcal{W}(\pi)} .
$$

In the appendix we clarify the relation between this definition and Weil's definition of the quadratic functional.

The Riemann hypothesis for $\Lambda(s, \pi)$ implies that the Weil scalar product is positive semidefinite on the test function vector space $\mathcal{A}$. To see this we note that $\rho=1-\bar{\rho}$ holds if and only if $\rho$ lies on the critical line $\Re(s)=\frac{1}{2}$. The Riemann hypothesis implies that for all $F(s) \in \mathcal{A}$,

$$
\langle F, F\rangle_{\mathcal{W}(\pi)}=\sum_{\rho \in Z(\pi)} F(\rho) \overline{F(1-\bar{\rho})}=\sum_{\rho \in Z(\pi)} F(\rho) \overline{F(\rho)}=\sum_{\rho \in Z(\pi)}|F(\rho)|^{2} \geq 0
$$

The Weil scalar product is not positive definite on the full class $\mathcal{A}$ since $F(s):=\xi(s, \pi) \in \mathcal{A}$ with $\langle F, F\rangle_{\mathcal{W}(\pi)}=0$. In the converse direction, Weil showed for various $L$-functions that the positive semidefiniteness of the Weil scalar product on suitable subsets of the test functions in $\mathcal{A}$. implies the Riemann Hypothesis for $\Lambda(s, \pi)$. A number of criteria of this sort are known, using different collections of test functions; essentially one needs that the test function set is sufficiently large to separate all the zeros $\rho=\rho_{\pi}$ (counting multiplicities).

\footnotetext{
${ }^{3}$ The region $|\Im(s)| \leq 1$ is left out here solely to avoid the points $s=0$ and $s=1$, because every nonzero element in $\mathcal{L}$ necessarily has a pole at one or both of these points.
} 
We define the Li class $\mathcal{L}$ of test functions to be the set of rational functions in the function field $\mathbb{C}(s)$ that vanish at infinity (on the Riemann sphere) and whose polar divisor is contained in the set $\{0,1\}$. The class $\mathcal{L}$ is closed under addition, multiplication and scalar multiplication, but does not contain the constant functions. A vector space basis for $\mathcal{L}$ consists of $\left\{\frac{1}{s^{n}}: n \geq 1\right\}$ and $\left\{\frac{1}{(1-s)^{n}}: n \geq 1\right\}$.

The special test functions $G_{n}(s) \in \mathcal{L}$ corresponding to the Li coefficients are

$$
G_{n}(s):=1-\left(1-\frac{1}{s}\right)^{n} \quad \text { for } \quad n \in \mathbb{Z} .
$$

The set of all $G_{n}(s)$, excluding $G_{0}(s) \equiv 0$, forms a vector space basis of $\mathcal{L}$. Indeed the change-of-basis matrix $G_{n}(s)(n \geq 1)$ relating these functions to $\left\{\frac{1}{s^{n}}: n \geq 1\right\}$ is an upper triangular unipotent matrix, and similarly for $G_{-n}(s)(n \geq 1)$ and $\left\{\frac{1}{(1-s)^{n}}: n \geq 1\right\}$. The class $\mathcal{L}$ is contained in $\mathcal{A}$, because the vanishing condition at $\infty$ implies a bound $F(s)=O\left(\frac{1}{|s|}\right)$ uniformly in the region $|\Im(s)| \geq 1$. Note that the class $\mathcal{L}$ has the property that every nonzero member of it has a pole at either $s=0$ or $s=1$, or both.

The following result computes the Weil scalar product with respect to this basis.

Theorem 3.1. Let $\pi$ be an irreducible cuspidal unitary automorphic representation of $G L(N)$, with associated $\xi$-function $\xi(s, \pi)$ and with Weil scalar product $\langle\cdot, \cdot \cdot\rangle_{\mathcal{W}(\pi)}$. For the Li test functions $G_{n}(s)=1-\left(1-\frac{1}{s}\right)^{n}$ there holds

$$
\left\langle G_{n}, G_{m}\right\rangle_{\mathcal{W}(\pi)}=\lambda_{n}(\pi)+\lambda_{-m}(\pi)-\lambda_{n-m}(\pi) .
$$

In particular

$$
\left\|G_{n}\right\|_{\mathcal{W}(\pi)}^{2}=\lambda_{n}(\pi)+\lambda_{-n}(\pi)=2 \Re\left(\lambda_{n}(\pi)\right) .
$$

Proof. By definition

$$
\lambda_{n}(\pi)=\sum_{\rho \in Z(\pi)}{ }^{\prime} G_{n}(\rho) .
$$

Since each function $G_{n}(s)$ is real on the real axis, the reflection principle gives

$$
\overline{G_{n}(1-\bar{\rho})}=G_{n}(1-\rho) \text {. }
$$

We also have the identity

$$
G_{-m}(s)=1-\left(1-\frac{1}{s}\right)^{-m}=1-\left(1-\frac{1}{s-1}\right)^{m}=G_{m}(1-s)
$$

and

$$
G_{n}(s) G_{-m}(s)=G_{n}(s)+G_{-m}(s)-G_{n-m}(s) .
$$

Combining all these gives

$$
\begin{aligned}
\left\langle G_{n}, G_{m}\right\rangle_{\mathcal{W}(\pi)} & =\sum_{\rho \in Z \pi} G_{n}(\rho) G_{m}(1-\rho)=\sum_{\rho} G_{n}(\rho) G_{m}(\rho) \\
& =\sum_{\rho \in Z(\pi)}{ }^{\prime}\left(G_{n}(\rho)+G_{-m}(\rho)-G_{n-m}(\rho)\right) \\
& =\sum_{\rho \in Z(\pi)}{ }^{\prime} G_{n}(\rho)+\sum_{\rho \in Z(\pi)}{ }^{\prime} G_{m}(\rho)+\sum_{\rho \in Z(\pi)}{ }^{\prime} G_{m}(\rho) \\
& =\lambda_{n}(\pi)+\lambda_{-m}(\pi)-\lambda_{n-m}(\pi) .
\end{aligned}
$$


the required result.

The Li class $\mathcal{L}$ is large enough to characterize the Riemann hypothesis in terms of the semidefiniteness of the Weil scalar product. Theorem 3.1 shows that semidefiniteness implies that all $\Re\left(\lambda_{n}\right) \geq 0$. The Riemann hypothesis then follows from Theorem 2.2 (2).

If the Riemann hypothesis holds, then the Weil scalar product is positive definite on $\mathcal{L}$. For it $F(x) \in \mathcal{L}$ is nonzero, then since it is a rational function it has finitely many zeros, so cannot vanish at all points of $Z(\pi)$. Thus

$$
\|F\|_{\mathcal{W}(\pi)}^{2}=\sum_{\rho \in Z(\pi)}|F(\rho)|^{2}>0 .
$$

The vector space $\mathcal{L}$ is then a pre-Hilbert space with this Hermitian scalar product, so it can be completed to a Hilbert space $\mathcal{H}_{\mathcal{L}}(\pi)$.

\section{Arithmetic Formula for Li Coefficients}

We relate the Li coefficients to various sums of the form $\sum_{\rho \in Z(\pi)}^{\prime} f(\rho)$ for various functions $f(s)$. In particular we split $\lambda_{n}(\pi)$ into two parts $S_{\infty}(n, \pi)$ and $S_{f}(n, \pi)$ given in Lemma 4.2 below.

The Li coefficients are expressible in terms of Laurent series coefficients around the point $s=1$ of of the logarithmic derivative of the $\xi$-function $\xi(s, \pi)$. We begin by relating these coefficients in terms of zeros.

Lemma 4.1. The power series expansion around $s=1$ of $\frac{\xi^{\prime}}{\xi}(s, \pi)$ is

$$
\frac{\xi^{\prime}}{\xi}(s+1, \pi)=\sum_{n=0}^{\infty}(-1)^{n} \sigma_{n+1}\left(\pi^{\vee}\right) s^{n} .
$$

in which the power sum $\sigma_{n}\left(\pi^{\vee}\right)=\sum_{\rho \in Z\left(\pi^{\vee}\right)}^{\prime} \frac{1}{\rho^{n}}$.

Proof. Since $\xi(s, \pi)$ is entire of order one we have the Hadamard product expansion

$$
\xi(s, \pi)=e^{A(\pi) s+B(\pi)} \prod_{\rho \in Z(\pi)}\left(1-\frac{s}{\rho}\right) e^{\frac{s}{\rho}} .
$$

Taking logarithms yields,

$$
\begin{aligned}
\log \xi(s, \pi) & =A(\pi) s+B(\pi)+\sum_{\rho \in Z(\pi)}\left(\log \left(1-\frac{s}{\rho}\right)+\frac{s}{\rho}\right) \\
& =A(\pi) s+B(\pi)-\sum_{\rho \in Z(\pi)}\left(\sum_{n=2}^{\infty} \frac{1}{n} \frac{s^{n}}{\rho^{n}}\right) \\
& =A(\pi) s+B(\pi)-\sum_{n=2}^{\infty} \sigma_{n}(\pi) \frac{s^{n}}{n} .
\end{aligned}
$$

One can also deduce by a $*$-convergent rearrangement that

$$
A(\pi)=-\sum_{\rho \in Z(\pi)}{ }^{\prime} \frac{1}{\rho}=-\sigma_{1}(\pi) .
$$


Differentiating yields

$$
-\frac{\xi^{\prime}}{\xi}(s, \pi)=\sum_{n=0}^{\infty} \sigma_{n+1}(\pi) s^{n}
$$

The functional equation (2.13) gives $\xi^{\prime}(s, \pi)=(-1)^{k+1} \xi^{\prime}\left(1-s, \pi^{\vee}\right)$, from which we obtain

$$
\frac{\xi^{\prime}}{\xi}(s+1, \pi)=-\frac{\xi^{\prime}}{\xi}\left(-s, \pi^{\vee}\right)=\sum_{n=0}^{\infty}(-1)^{n} \sigma_{n+1}\left(\pi^{\vee}\right) s^{n},
$$

as required.

We now write

$$
\frac{\xi^{\prime}}{\xi}(s+1, \pi)=\left(\frac{1}{2} \log Q(\pi)+\sum_{j=1}^{N} \frac{\Gamma_{\mathbb{R}}^{\prime}}{\Gamma_{\mathbb{R}}}\left(s+1+\kappa_{j}(\pi)\right)\right)+\frac{L^{\prime}}{L}(s+1, \pi)-\frac{e(0, \pi)}{s+1}-\frac{e(1, \pi)}{s}
$$

We define Laurent series coefficients around $s=1$ of the first two terms on the right, as follows. The coefficents $\left\{\tau_{n}(\pi): n \geq 1\right\}$ are defined by

$$
\sum_{n=0}^{\infty} \tau_{n}(\pi) s^{j}:=\frac{1}{2} \log Q(\pi)+\sum_{j=1}^{N} \frac{\Gamma_{\mathbb{R}}^{\prime}}{\Gamma_{\mathbb{R}}}\left(s+1+\kappa_{j}(\pi)\right)
$$

in which we have using (2.3) that

$$
\frac{\Gamma_{\mathbb{R}}^{\prime}}{\Gamma_{\mathbb{R}}}(s)=-\frac{1}{2} \log \pi+\frac{1}{2} \frac{\Gamma^{\prime}}{\Gamma}\left(\frac{s}{2}\right)
$$

The coefficients $\tau_{n}(\pi)$ are real-valued, as a consequence of Theorem [2.1(2). Next, the coefficients $\left\{\eta_{n}(\pi): n \geq 1\right\}$ are defined by

$$
\sum_{n=0}^{\infty} \eta_{n}(\pi) s^{n}:=-\frac{L^{\prime}}{L}(s+1, \pi)-\frac{\delta(\pi)}{s}
$$

where $\delta(\pi)=1$ if $\pi=\pi_{\text {triv }}$ and $\delta(\pi)=0$ otherwise. (The minus sign is present to agree with the convention in [4, eqn. (4.3)].) For $\pi=\pi_{\text {triv }}$ these are the coefficients $\eta_{n}$ given in [4].

Lemma 4.2. Let $\pi$ be an irreducible cuspidal automorphic representation on $G L(N)$ over $\mathbb{Q}$. Then for all $n \geq 1$,

$$
\lambda_{n}(\pi)=S_{\infty}(n, \pi)-S_{f}\left(n, \pi^{\vee}\right)+\delta(\pi),
$$

in which

$$
S_{\infty}\left(n, \pi^{\vee}\right)=\sum_{j=1}^{n}\left(\begin{array}{l}
n \\
j
\end{array}\right) \tau_{j-1}\left(\pi^{\vee}\right)
$$

and

$$
S_{f}\left(n, \pi^{\vee}\right)=\sum_{j=1}^{n}\left(\begin{array}{l}
n \\
j
\end{array}\right) \eta_{j-1}\left(\pi^{\vee}\right),
$$

and $\delta(\pi)=1$ if $\pi=\pi_{\text {triv }}$ and $\delta(\pi)=0$ otherwise. 
Remark. $\quad$ Note that $S_{\infty}(n, \pi)=S_{\infty}\left(n, \pi^{\vee}\right)$ and $\delta(\pi)=\delta\left(\pi^{\vee}\right.$. Also, since $\lambda_{-n}(\pi)=\overline{\lambda_{n}(\pi)}=$ $\lambda_{n}\left(\pi^{\vee}\right)$, we obtain from (4.6) applied to $\pi^{\vee}$ that

$$
\lambda_{-n}(\pi)=S_{\infty}(n, \pi)-S_{f}(n, \pi)+\delta(\pi) .
$$

Proof. Comparison of Lemma 4.1 for $\pi^{\vee}$ and the formula (4.2) yields

$$
(-1)^{j} \sigma_{j+1}(\pi)=\tau_{j}\left(\pi^{\vee}\right)-\eta_{j}\left(\pi^{\vee}\right)+(-1)^{j} \delta\left(\pi^{\vee}\right)
$$

Substituting this formula into (2.18), and using $-e(0, \pi)=\delta(\pi)=\delta\left(\pi^{\vee}\right)$, we obtain the desired result.

In the expression (4.6) the term $S_{\infty}\left(n, \pi^{\vee}\right)$ corresponds to the contribution of the archimedean primes and $S_{f}\left(n, \pi^{\vee}\right)$ corresponds to the finite primes. There are also extra contributions from singularities at $s=0$ and $s=1$. The $s=1$ contribution cancels against the singularity at $s=1$ of $\frac{L^{\prime}}{L}(s, \pi)$, but the $s=0$ singularity remains and contributes the constant $-e(0, \pi) \delta\left(\pi^{\vee}\right)$.

For $\pi_{\text {triv }}=\pi_{\text {triv }}^{\vee}$ on $G L(1)$ the terms $S_{\infty}(n, \pi)$ and $S_{f}(n, \pi)$ form parts of the arithmetic expression for the Li coefficients $\lambda_{n}$ given in Theorem 2 of [4]. We have $\mu_{1}\left(\pi_{\text {triv }}\right)=0$ and

$$
S_{\infty}\left(n, \pi_{t r i v}\right)=-\sum_{j=1}^{n}(-1)^{j+1}\left(\begin{array}{l}
n \\
j
\end{array}\right)\left(1-\frac{1}{2^{j}}\right) \zeta^{*}(j),
$$

in which $\zeta^{*}(j)=\zeta(j)$ for $j \geq 2$, and $\zeta^{*}(1)=\log (4 \pi)+\gamma$, where $\gamma$ is Euler's constant. For the other term, writing

$$
-\frac{\zeta^{\prime}}{\zeta}(s+1)=\frac{1}{s}+\sum_{j=0}^{\infty} \eta_{j} s^{j}
$$

we have

$$
S_{f}\left(n, \pi_{\text {triv }}\right)=\sum_{j=1}^{n}(-1)^{j-1}\left(\begin{array}{l}
n \\
j
\end{array}\right) \eta_{j-1} .
$$

In [4] it is shown that the coefficients $\eta_{j}$ are given by

$$
\eta_{j}:=\frac{(-1)^{j}}{j !} \lim _{N \rightarrow \infty}\left(\sum_{m=1}^{N} \frac{\Lambda(m)(\log m)^{j}}{m}-\frac{1}{j+1} \frac{N}{(\log N)^{j+1}}\right),
$$

in which $\Lambda(m)$ is the von Mangoldt function.

There exist formal arithmetic expressions for $S_{f}(n, \pi)$ in the general case that are similar in spririt to (4.13). Logarithmically differentiating the Euler product for $L(s, \pi)$ in the region $\Re(s)>1$ yields

$$
-\frac{L^{\prime}}{L}(s, \pi)=\sum_{n=1}^{\infty} \Lambda_{\pi}(n) n^{-s}
$$

in which for $n=p^{m}$ a prime power,

$$
\Lambda_{\pi}\left(p^{m}\right)=\frac{1}{m} \sum_{k=1}^{N}\left(\alpha_{k, p}(\pi)\right)^{m}
$$


and $\Lambda_{\pi}(n)=0$ otherwise. Now for all $\pi \neq \pi_{\text {triv }}$ using (4.5) (which is now analytic at $s=1$ ) we have

$$
\eta_{j}(\pi)=-\left.\frac{1}{j !} \frac{d^{j}}{d s^{j}}\left[\frac{L^{\prime}}{L}(s, \pi)\right]\right|_{s=1}
$$

Using (4.14) we obtain, formally,

$$
\eta_{j}(\pi) \simeq \frac{(-1)^{j}}{j !} \sum_{m=1}^{\infty} \frac{\Lambda_{\pi}(m)(\log m)^{j}}{m}
$$

The sum on the right at best converges conditionally, when viewed as $\lim _{T \rightarrow \infty} \sum_{n \leq T}$. The conditional convergence is known to hold for $L$-functions on $G L(1)$. One can show

$$
\eta_{j}(\pi)=\lim _{s \searrow 1} \frac{(-1)^{j}}{j !} \sum_{m=1}^{\infty} \frac{\Lambda_{\pi}(m)(\log m)^{j}}{m^{s}}
$$

with $s \searrow 1$ along the real axis. Explicit expressions for $\eta_{j}(\pi)$ for various $L$-functions and modular forms are derived in X. Li [35, 36, 37.

There exist simplified formulas for the coefficients $\tau_{n}(\pi)$ analogous to (4.11), which involve values of the Hurwitz zeta function $\zeta(s, z)=\sum_{j=0}^{\infty} \frac{1}{(n+z)^{s}}$ with $\Re(z)>0$.

Lemma 4.3. For an irreducible cuspidal automorphic representation $\pi$ on $G L(N)$ there holds, for $n \geq 1$,

$$
\tau_{n}(\pi)=\left(-\frac{1}{2}\right)^{n+1} \sum_{j=1}^{n} \zeta\left(n+1, \frac{\kappa_{j}(\pi)+1}{2}\right) .
$$

In addition

$$
\tau_{0}(\pi)=\frac{1}{2} \log Q(\pi)-\frac{N}{2} \log \pi+\frac{1}{2} \sum_{j=1}^{N} \frac{\Gamma^{\prime}}{\Gamma}\left(\frac{\kappa_{j}(\pi)-1}{2}\right) .
$$

where $\gamma$ is Euler's constant.

Proof. We recall that $\Gamma_{\mathbb{R}}(s)=\pi^{-\frac{s}{2}} \Gamma\left(\frac{s}{2}\right)$., so that

$$
\frac{\Gamma_{\mathbb{R}}^{\prime}}{\Gamma_{\mathbb{R}}}(s)=-\frac{1}{2} \log \pi+\frac{1}{2} \frac{\Gamma^{\prime}}{\Gamma}\left(\frac{s}{2}\right) .
$$

The digamma function $\psi(s)=\frac{\Gamma^{\prime}}{\Gamma}(s)$ has the partial fraction expansion

$$
\psi(s+1)=\frac{\Gamma^{\prime}}{\Gamma}(s+1)=-\gamma+\sum_{m=1}^{\infty}\left(\frac{1}{m}-\frac{1}{s+m}\right),
$$

in which $\gamma \approx 0.5771$ is Euler's constant. For $\Re(z)>-1$ we define the power series coefficients $\left\{\tilde{\tau}_{n}(z): n \geq 0\right\}$ by

$$
\sum_{n=0}^{\infty} \tilde{\tau}_{n}(z) s^{n}:=\frac{\Gamma^{\prime}}{\Gamma}(s+1+z)
$$

Here

$$
\tilde{\tau}_{n}(z)=\left.\frac{1}{n !} \frac{d^{n}}{d s^{n}}\left(\frac{\Gamma^{\prime}}{\Gamma}(s+1+z)\right)\right|_{s=0}
$$


We have

$$
\tilde{\tau}_{0}(z)=-\gamma+\sum_{m=1}^{\infty} \frac{z}{m(m+z)}
$$

and, for $n \geq 1$,

$$
\tilde{\tau}_{n}(z)=(-1)^{n+1} \sum_{m=1}^{\infty}\left(\frac{1}{m+z}\right)^{n+1},
$$

which follows by differentiating the partial fraction expansion (4.19) at $s=z+1$. This is essentially a special value of the Hurwitz zeta function, for $n \geq 1$,

$$
\tilde{\tau}_{n}(z)=(-1)^{n+1} \zeta(n+1, z+1) .
$$

Comparing power series coefficients of the definition (4.3) with those of (4.18) yields, for $n \geq 1$,

$$
\tau_{n}(\pi)=\frac{1}{2} \sum_{j=1}^{N} \tilde{\tau}_{n}\left(\frac{\kappa_{j}(\pi)-1}{2}\right)\left(\frac{1}{2}\right)^{n},
$$

and using (4.23) then yields (4.16).

Finally, the case $n=0$ follows from (4.3) for $n=0$ using (4.21).

Remark. For the case $\pi_{\text {triv }}$ on $G L(1)$ Lemma 4.3 yields

$$
\begin{aligned}
\tau_{n}\left(\pi_{\text {triv }}\right) & =\left(-\frac{1}{2}\right)^{n+1} \zeta\left(n+1, \frac{1}{2}\right) \\
& =\left(-\frac{1}{2}\right)^{n+1} \sum_{m=0}^{\infty} \frac{1}{\left(m+\frac{1}{2}\right)^{n+1}} \\
& =(-1)^{n+1} \sum_{m=0}^{\infty}\left(\frac{1}{2 m+1}\right)^{n+1}=(-1)^{n+1}\left(1-\frac{1}{2^{n+1}}\right) \zeta(n+1),
\end{aligned}
$$

and with further work one can deduce

$$
\tau_{0}\left(\pi_{\text {triv }}\right)=-\left(\frac{1}{2}\right)(\log (4 \pi)+\gamma)
$$

from which one can deduce (4.11).

\section{Bounds for $S_{\infty}(n, \pi)$}

In this and the next section we consider the order of growth of the terms $S_{\infty}(n, \pi)$ and $S_{f}(n, \pi)$, respectively. Here we we obtain the following unconditional result for $S_{\infty}(n, \pi)$.

Theorem 5.1. For any irreducible cuspidal (unitary) automorphic representation $\pi$ on $G L(N)$ the quantities $S_{\infty}(n, \pi)$ are real-valued. There is a constant $K(\pi)$ such that for $n \geq K(\pi)$ there holds

$$
S_{\infty}(n, \pi)=\left(\frac{N}{2}\right) n \log n+C_{1}(\pi) n+O(N(K(\pi)+1)) .
$$

Here

$$
C_{1}(\pi)=\frac{N}{2}(\gamma-1-\log (2 \pi))+\frac{1}{2} \log Q(\pi)
$$


where $\gamma$ is Euler's constant. One can take

$$
K(\pi)=\max _{1 \leq j \leq N}\left|\kappa_{j}(\pi)\right|^{2}
$$

and the implied constant in the O-notation is absolute.

Note that $\left.C_{(} \pi\right)=C_{1}\left(\pi^{\vee}\right)$, since $Q(\pi)=Q\left(\pi^{\vee}\right)$, and that $C_{1}(\pi)$ does not depend on the archimedean parameters $\left\{\kappa_{j}(\pi): 1 \leq j \leq N\right\}$, though these values appear in the analysis. For the Li coefficients we have

$$
C_{1}\left(\pi_{\text {triv }}\right)=\frac{1}{2}(\gamma-1-\log (2 \pi)) \simeq-1.1303307 .
$$

using $Q\left(\pi_{\text {triv }}\right)=1$.

To begin the proof we introduce the quantities

$$
T(n, z):=\sum_{j=1}^{n}\left(\begin{array}{l}
n \\
j
\end{array}\right)\left(\frac{1}{2}\right)^{j} \tilde{\tau}_{j-1}(z) .
$$

The formula for $S_{\infty}(n, \pi)$ in Lemma 4.2 can be expressed in terms of these for various values of $z$. Indeed we obtain, for $n \geq 1$,

$$
S_{\infty}(n, \pi)=\sum_{j=1}^{N} T\left(n, \frac{\kappa_{j}\left(\pi^{\vee}\right)-1}{2}\right)+\left(\begin{array}{l}
n \\
1
\end{array}\right)\left(\frac{1}{2} \log Q(\pi)-\frac{N}{2} \log \pi\right),
$$

using (4.24). We proceed to estimate an individual sum $T(n, z)$. The formulas for $\tilde{\tau}_{j}(z)$ give

$$
T(n, z)=T_{1}(n, z)+T_{2}(n, z)
$$

in which

$$
T_{1}(n, z)=\frac{n}{2} \frac{\Gamma^{\prime}}{\Gamma}(1+z)=\left(\begin{array}{c}
n \\
1
\end{array}\right) \frac{1}{2}\left(-\gamma+\sum_{m=1}^{\infty} \frac{z}{m(m+z)}\right) .
$$

and

$$
T_{2}(n, z):=\sum_{j=2}^{n}\left(\begin{array}{l}
n \\
j
\end{array}\right) \frac{1}{2^{j}}\left((-1)^{j} \sum_{m=1}^{\infty} \frac{1}{(m+z)^{j}}\right) .
$$

The second sum converges absolutely and can be rearranged as

$$
\begin{aligned}
T_{2}(n, z) & =\sum_{m=1}^{\infty} \sum_{j=2}^{n}\left(\begin{array}{l}
n \\
j
\end{array}\right)\left(-\frac{1}{2}\right)^{j} \frac{1}{(m+z)^{j}} \\
& =\sum_{m=1}^{\infty}\left(\left(1-\frac{1}{2 m+2 z}\right)^{n}-1+\frac{n}{2 m+2 z}\right) .
\end{aligned}
$$

We divide this sum into two parts

$$
T_{2}(n, z)=T_{20}(n, z)+T_{21}(n, z)
$$

by splitting the summation range from $m=[1, n]$ and $m=[n+1, \infty)$ respectively, and treat these in the following two lemmas. 
Lemma 5.1. For any complex number $z$ with $\Re(z) \geq-\frac{3}{4}$, the quantity

$$
T_{20}(n, z)=\sum_{m=1}^{n}\left(\left(1-\frac{1}{2 m+2 z}\right)^{n}-1+\frac{n}{2 m+2 z}\right)
$$

satisfies, for all $n \geq|z|^{2}$,

$$
T_{20}(n, z)=\frac{1}{2}(n \log n)+\left(-\frac{1}{2} \frac{\Gamma^{\prime}}{\Gamma}(1+z)+\beta_{\infty}+\frac{1}{\sqrt{e}}-1\right) n+O(|z|+1) .
$$

in which

$$
\beta_{\infty}=\int_{1}^{\infty} e^{-\frac{t}{2}} \frac{d t}{t} \simeq 0.559774
$$

Proof. The sum (5.10) consists of three subsums, from each of its terms. The middle subsum in (5.10) gives $-n$. For the third subsum,

$$
\begin{aligned}
K(n, z)=\sum_{m=1}^{n} \frac{n}{2 m+2 z} & =\frac{n}{2} \sum_{m=1}^{n}\left(\frac{1}{m}-\frac{z}{m(m+z)}\right) \\
& =\frac{n}{2}\left(\log n+\gamma+O\left(\frac{1}{n}\right)\right)-\frac{n}{2}\left(\frac{\Gamma^{\prime}}{\Gamma}(1+z)+\gamma+O\left(\frac{|z|+1}{n}\right)\right) \\
& =\frac{1}{2} n \log n-\frac{n}{2} \frac{\Gamma^{\prime}}{\Gamma}(1+z)+O(|z|+1)
\end{aligned}
$$

in which we used the partial fraction formula for the digamma function and a bound for the error in truncating it at the $n$-th term.

We now consider the first subsum in (5.10), call it

$$
J(n, z):=\sum_{m=1}^{n}\left(1-\frac{1}{2 m+2 z}\right)^{n} .
$$

Setting $z=x+i y$, for real $t \geq 1$ we have

$$
\left|1-\frac{1}{2 t+2 z}\right|^{2}=1+\frac{1-4(t+x)}{4\left((t+x)^{2}+y^{2}\right)},
$$

The condition $\Re(z) \geq-\frac{3}{4}$ now implies that $\left|1-\frac{1}{2 t+2 z}\right|^{2} \leq 1$, so that each term above is $O(1)$. We approximate the sum by an integral, and assert that for $n \geq|z|^{2}$ there holds

$$
J(n, z)=\int_{1}^{n}\left(1-\frac{1}{2 t+2 z}\right)^{n} d t+O(|z|+1) .
$$

with a constant independent of $z$. To establish this is we show that both sum and integral separately contribute $O(1)$ over the range $1 \leq t \leq n^{\frac{2}{3}}$, and then show on the remaining range $n^{\frac{2}{3}} \leq t \leq n$ that their difference is $O(1)$. On the initial range we use $1-x \leq e^{-x}$ for $0 \leq x \leq 1$ to get from (5.14) that, for $t \geq 3$,

$$
\begin{aligned}
\left|1-\frac{1}{2 t+2 z}\right|^{n} & \leq\left(1+\frac{1-4(t+x)}{4\left((t+x)^{2}+y^{2}\right)}\right)^{\frac{n}{2}} \\
& \leq \exp \left(\frac{1-4(t+x)}{4\left((t+x)^{2}+y^{2}\right)} \frac{n}{2}\right) \\
& \leq \exp \left(-\frac{(t+x)}{(t+x)^{2}+y^{2}} \frac{n}{4}\right) .
\end{aligned}
$$


Now the function $f(w)=\frac{w}{w^{2}+y^{2}}$ is nonnegative on the positive real axis, increasing to a maximum at $w=y$ and decreasing monotonically thereafter. Applied to the exponential above, we find the the terms for integer $t=m$ with $y \leq m \leq n^{\frac{2}{3}}$ have negative exponent decreasing in absolute value, so the largest term occurs at the top endpoint, and is bounded by $\exp \left(-c n^{-\frac{1}{3}}\right)$, so the sum over all these terms is $O(1)$. The first $y$ terms each contribute $O(1)$, giving $O(|z|+1)$ in all. This bounds the sum, and bounding the integral over this range is similar.

On the remaining range $n^{\frac{2}{3}} \leq m \leq 1$ with $m \leq t \leq m+1$ we have

$$
\begin{aligned}
\left(1-\frac{1}{2 t+2 z}\right)^{n} & =\left(1-\frac{1}{2 m+2 z}\right)^{n}\left(\frac{1-\frac{1}{2 m+2 z}}{1-\frac{1}{2 t+2 z}}\right)^{n} \\
& =\left(1-\frac{1}{2 m+2 z}\right)^{n}\left(1+O\left(\frac{1}{|2 t+2 z|^{2}}\right)\right)^{n} \\
& =\left(1-\frac{1}{2 m+2 z}\right)^{n}\left(1+O\left(\frac{n}{|2 t+2 z|^{2}}\right)\right),
\end{aligned}
$$

where we used $|2 t+2 z|^{2} \geq 2 n$. Thus, for $n \geq|z|^{2}$, the error between the sum and the integral is bounded by an absolute constant times

$$
\int_{n^{2 / 3}}^{n}\left(1-\frac{1}{2 t+2 z}\right)^{n} \frac{n}{|2 t+2 z|^{2}} d t \leq O(1) .
$$

This last estimate is obtained by observing that the integrand is maximized (in absolute value) at its top endpoint, where it is $O\left(\frac{1}{n}\right)$. We conclude that (5.15) holds.

We now integrate by parts on the right side of (5.15) to obtain

$$
J(n, z)=J_{1}(n, z)-J_{2}(n, z)+O(|z|+1),
$$

in which

$$
J_{1}(n, z):=\left.t\left(1-\frac{1}{2 t+2 z}\right)^{n}\right|_{t=1} ^{n}=n\left(1-\frac{1}{2 n+2 z}\right)^{n}-\left(1-\frac{1}{2+2 z}\right)^{n}
$$

and

$$
J_{2}(n, z)=n \int_{1}^{n} \frac{2 t}{(2 t+2 z)^{2}}\left(1-\frac{1}{2 t+2 z}\right)^{n-1} d t .
$$

The first term on the right side is estimated for $n>|z|^{2}$ by

$$
J_{1}(n, z)=n \exp \left(-\frac{n}{2 n+2 z}\right)\left(1+O\left(\frac{|z|+1}{n}\right)+O(1)=\frac{1}{\sqrt{e}} n+O(|z|+1),\right.
$$

For the remaining integral $J_{2}(n, z)$, we show that $\tilde{J}_{2}(n, z)=\frac{1}{n} J_{2}(n, z)$ approaches a limit as $n \rightarrow \infty$, using the identity $\left(1-\frac{1}{u}\right)^{u}$ approaches $e$ as $\Re(u) \rightarrow \infty$. We rescale it with the variable change $u=n t$ to obtain

$$
\begin{aligned}
\tilde{J}_{2}(n, z) & =\int_{\frac{1}{n}}^{1} \frac{2 n u}{(2 n u+z)^{2}}\left(1-\frac{1}{2(n u+z)}\right)^{n-1} n d u \\
& =\frac{1}{2} \int_{\frac{1}{2 n}}^{1} \frac{u}{\left(u+\frac{2 z}{n}\right)^{2}}\left[\left(1-\frac{1}{2(n u+z)}\right)^{2(n u+z)}\right]^{\frac{n-1}{2(n u+z)}} d u \\
& =\frac{1}{2} \int_{\frac{1}{n}}^{1} \frac{1}{u} e^{-\frac{1}{2 u}} d u+O\left(\frac{|z|+1}{n}\right) \\
& =\frac{1}{2} \int_{0}^{1} \frac{1}{u} e^{-\frac{1}{2 u}} d u+O\left(\frac{|z|+1}{n}\right)
\end{aligned}
$$


Now we set

$$
\beta_{\infty}:=\int_{0}^{1} e^{-\frac{1}{2 u}} \frac{d u}{u}=\int_{1}^{\infty} e^{-\frac{t}{2}} \frac{d t}{t}
$$

and we have obtained

$$
J_{2}(n, z)=\frac{1}{2} \beta_{\infty} n+O(|z|+1) .
$$

Combining all these estimates gives, for $n \geq|z|^{2}$,

$$
\begin{aligned}
T_{20}(n, z) & =J_{1}(n, z)-J_{2}(n, z)-n+K(n, z)+O(|z|+1) \\
& =(\sqrt{e}-1) n+\frac{1}{2} n \log n-\frac{n}{2} \frac{\Gamma^{\prime}}{\Gamma}(1+z)-\frac{1}{2} \beta_{\infty} n+O(|z|+1) \\
& =\frac{1}{2} n \log n+\left(-\frac{1}{2} \frac{\Gamma^{\prime}}{\Gamma}(1+z)-\frac{1}{2} \beta_{\infty}+\frac{1}{\sqrt{e}}-1\right) n+O(|z|+1),
\end{aligned}
$$

the desired estimate.

Lemma 5.2. For $\Re(z) \geq-1$ and $n \geq|z|+2$, the quantity

$$
T_{21}(n, z)=\sum_{m=n+1}^{\infty}\left(\left(1-\frac{1}{2 m+2 z}\right)^{n}-1+\frac{n}{2 m+2 z}\right)
$$

satisfies

$$
T_{21}(n, z)=\left(\frac{1}{2}-\frac{1}{\sqrt{e}}+\frac{1}{2} \alpha_{\infty}\right) n+O(|z|+1),
$$

in which

$$
\alpha_{\infty}=\int_{0}^{1}\left(1-e^{-\frac{1}{2} t}\right) \frac{d t}{t} \simeq 0.443842 .
$$

The implied O-constant is absolute.

Proof. In what follows we assume $n \geq|z|+2$. We approximate the sum by an integral

$$
T_{21}(n, z)=\int_{n}^{\infty}\left(\left(1-\frac{1}{2 t+2 z}\right)^{n}-1+\frac{n}{2 t+2 z}\right) d t+O(1) .
$$

To justify the error term, we observe that for $n \leq m \leq t \leq m+1$ there holds

$$
\left(1-\frac{1}{2 t+2 z}\right)^{n}=\left(1-\frac{1}{2 m+2 z}+O\left(\frac{1}{|(2 m+2 z)(2 t+2 z)|}\right)\right)^{n}=\left(1-\frac{1}{2 m+2 z}\right)^{n}\left(1+O\left(\frac{n}{(t+|z|)^{2}}\right)\right.
$$

where we used $\Re(z) \geq-1$ and $n \geq 2$. We also use

$$
\left|\frac{n}{t+z}-\frac{n}{m+z}\right| \leq\left|\frac{n(m-t)}{\mid(t+z)(m+z)}\right| \leq \frac{n}{(m+|z|-1)^{2}},
$$

Viewing the sum as an integral of a step function, we have bounded the difference between the integrands at $t$ by an absolute constant times $\frac{n}{(t+|z|-1)^{2}}$, and for $n \geq 3$ we have

$$
\int_{n}^{\infty} \frac{n}{(t+|z|-1)^{2}} d t \leq n \int_{n-1}^{\infty} \frac{1}{t^{2}} d t=O(1)
$$


and (5.22) follows.

Making the change of variable $u=\frac{1}{2 t+2 z}$ we obtain

$$
T_{21}(n, z)=\frac{1}{2} \int_{0}^{\frac{1}{2 n+2 z}}\left[(1-u)^{n}-1+n u\right] \frac{d u}{u^{2}}+O(1) .
$$

This integral is a contour integral (since $z$ is complex) but the answer is independent of the contour, since the integrand is analytic. An integration by parts yields $T_{21}(n, z)=K_{1}(n, z)-$ $K_{2}(n, z)+O(1)$ in which

$$
\begin{aligned}
K_{1}(n, z) & =-\left.\frac{1}{2 u}\left[(1-u)^{n}-1+n u\right]\right|_{u=0} ^{\frac{1}{2 n+2 z}} \\
& =-(n+z)\left(\left(1-\frac{1}{2 n+2 z}\right)^{n}-1+\frac{n}{2 n+2 z}\right),
\end{aligned}
$$

and

$$
K_{2}(n, z):=\frac{1}{2} \int_{0}^{\frac{1}{2 n+2 z}}\left[-n(1-u)^{n-1}+n\right] \frac{d u}{-u}=\frac{n}{2} \int_{0}^{\frac{1}{2 n+2 z}}\left[(1-u)^{n-1}-1\right] \frac{d u}{u} .
$$

To estimate $K_{1}(n, z)$ we write $z=x+i y$, with $x \geq-1$, to obtain

$$
1-\frac{1}{2 n+2 z}=\left(1-\frac{2 n+2 x}{4(n+x)^{2}+4 y^{2}}\right)+i \frac{2 y}{4(n+x)^{2}+4 y^{2}}=r e^{i \theta},
$$

in which $r=1-\frac{1}{2 n}+O\left(\frac{1}{n^{2}}\right)$ and $\theta=O\left(\frac{|z|+1}{n^{2}}\right)$. It follows that $r^{n} e^{i n \theta}=e^{-\frac{1}{2}}+O\left(\frac{|z|+1}{n}\right)$, and we obtain

$$
\begin{aligned}
K_{1}(n, z) & =-n\left[\left(1-\frac{1}{2 n+2 z}\right)^{n}-\frac{1}{2}\right]+O(|z|+1) \\
& =-n\left[r^{n} e^{i n \theta}-\frac{1}{2}\right]+O(|z|+1) \\
& =n\left(\frac{1}{2}-\frac{1}{\sqrt{e}}+O\left(\frac{|z|+1}{n}\right)\right)+O(|z|+1) .
\end{aligned}
$$

Expanding the integral $K_{2}(n, z)$ in powers of $u$ yields

$$
\begin{aligned}
K_{2}(n, z) & =\frac{n}{2}\left(\sum_{j=1}^{n} \int_{0}^{\frac{1}{2 n+2 z}}(-1)^{j}\left(\begin{array}{c}
n \\
j
\end{array}\right) u^{j-1} d u\right) \\
& =\frac{n}{2}\left(\sum_{j=1}^{n} \frac{(-1)^{j}}{j}\left(\begin{array}{c}
n \\
j
\end{array}\right)\left(\frac{1}{2 n+2 z}\right)^{j}\right) .
\end{aligned}
$$

We set

$$
\alpha_{n}(z)=\sum_{j=1}^{n} \frac{(-1)^{j}}{j}\left(\begin{array}{l}
n \\
j
\end{array}\right)\left(\frac{1}{2 n+2 z}\right)^{j}
$$

and

$$
\alpha_{\infty}:=\sum_{j=1}^{\infty} \frac{(-1)^{j+1}}{j} \frac{1}{j ! 2^{j}}=\int_{0}^{\frac{1}{2}}\left(1-e^{-v}\right) \frac{d v}{v}=\int_{0}^{1}\left(1-e^{-\frac{1}{2} t}\right) \frac{d t}{t} .
$$


We assert that for $\Re(z) \geq-1, n \geq 2$,

$$
\left|\alpha_{n}(z)+\alpha_{\infty}\right|=O\left(\frac{|z|+1}{n}\right) .
$$

where the constant in the O-notation is independent of $z$. Indeed we note that

$$
\left(\begin{array}{c}
n \\
j
\end{array}\right)\left(\frac{1}{2 n+2 z}\right)^{j}=\frac{1}{j ! 2^{j}}\left(\frac{n}{n+z}\right)\left(\frac{n-1}{n+z}\right) \cdots\left(\frac{n-j+1}{n+z}\right),
$$

which since $\Re(z) \geq-1$ yields

$$
\left|\left(\begin{array}{c}
n \\
j
\end{array}\right)\left(\frac{1}{2 n+2 z}\right)^{j}\right| \leq \frac{2}{j ! 2^{j}} .
$$

and also for $0 \leq j \leq 2 \log n$ that

$$
\left|\left(\begin{array}{c}
n \\
j
\end{array}\right)\left(\frac{1}{2 n+2 z}\right)^{j}-\frac{1}{j ! 2^{j}}\right|=O\left(\frac{|z|+1}{n}\right) .
$$

Combining these estimates with

$$
\left|\alpha_{\infty}+\sum_{j=1}^{2 \log n} \frac{(-1)^{j}}{j} \frac{1}{j ! 2^{j}}\right| \leq O\left(\frac{1}{n}\right),
$$

yields (5.29), so that $K_{2}(n, z)=-\frac{1}{2} \alpha_{\infty} n+O(|z|+1)$.

Combining all the estimates above gives

$$
\begin{aligned}
T_{21}(n, z) & \left.=K_{1}(n, z)-K_{2}(n, z)+O(1)\right) \\
& =\left(\frac{1}{2}-\frac{1}{\sqrt{e}}+\frac{1}{2} \alpha_{\infty}\right) n+O(|z|+1),
\end{aligned}
$$

the desired estimate.

\section{Proof of Theorem 5.1, Lemma 4.2 gives}

$$
\begin{aligned}
S_{\infty}(n, \pi) & =\sum_{j=1}^{n}\left(\begin{array}{l}
n \\
j
\end{array}\right) \tau_{j-1}(\pi) \\
& =\left(\sum_{k=1}^{N} \sum_{j=1}^{n}\left(\begin{array}{c}
n \\
j
\end{array}\right)(-1)^{j} \tilde{\tau}_{j-1}\left(\frac{\kappa_{j}(\pi)-1}{2}\right)\left(\frac{1}{2}\right)^{j}\right)+\left(\begin{array}{l}
n \\
1
\end{array}\right)\left(\frac{1}{2} \log Q(\pi)-\frac{N}{2} \log \pi\right) \\
& =\left(\sum_{k=1}^{N} T\left(n, \frac{\kappa_{k}(\pi)-1}{2}\right)\right)+\left(\frac{1}{2} \log Q(\pi)-\frac{N}{2} \log \pi\right) n \\
& =\left(\frac{1}{2} \log Q(\pi)-\frac{N}{2} \log \pi+\frac{1}{2} \sum_{j=1}^{N} \frac{\Gamma^{\prime}}{\Gamma}\left(\frac{\kappa_{k}(\pi)+1}{2}\right)\right) n+\sum_{k=1}^{N} T_{2}\left(n, \frac{\kappa_{k}(\pi)-1}{2}\right) .
\end{aligned}
$$

where we made use of the decomposition $T(n, z)=T_{1}(n, z)+T_{2}(n, z)$ and the formula (5.7) for $T_{1}(n, z)$. 
Now we suppose that $n \geq K(\pi):=\max \left\{\left|\kappa_{k}(\pi)\right|^{2}: 1 \leq k \leq N\right\}$ and note by Theorem 2.1 (2) that each $\Re\left(\frac{\kappa_{k}(\pi)+1}{2}\right)>-\frac{3}{4}$, so that we can apply Lemma 5.1 and 5.2 We obtain

$$
\begin{aligned}
T_{2}\left(n, \frac{\kappa_{k}(\pi)-1}{2}\right)= & \frac{1}{2} n \log n+\left(-\frac{1}{2} \frac{\Gamma^{\prime}}{\Gamma}\left(\frac{\kappa_{k}(\pi)+1}{2}\right)+\frac{1}{\sqrt{e}}-1\right) n \\
& \left.+\left(\frac{1}{2}-\frac{1}{\sqrt{e}}+\frac{1}{2} \alpha_{\infty}-\frac{1}{2} \beta_{\infty}\right) n+O(K(\pi)+1)\right) \\
= & \frac{1}{2} n \log n+\left(-\frac{1}{2} \frac{\Gamma^{\prime}}{\Gamma}\left(\frac{\kappa_{k}(\pi)+1}{2}\right)+\frac{1}{2}\left(\alpha_{\infty}-\beta_{\infty}-1\right)\right) n+O(K(\pi)+1) .
\end{aligned}
$$

Substituting this in the previous formula yields

$$
\begin{aligned}
S_{\infty}(n, \pi)=\left(\frac{N}{2}\right) & n \log n+\left(\frac{N}{2}\left(\alpha_{\infty}-\beta_{\infty}-1-\log \pi\right)+\frac{1}{2} \log Q(\pi)\right) n \\
& +O(N(K(\pi)+1)) .
\end{aligned}
$$

To complete the proof we must establish the identity ${ }^{4}$

$$
\alpha_{\infty}-\beta_{\infty}=\gamma-\log 2
$$

where $\gamma$ is Euler's constant. We start from

$$
\alpha_{\infty}-\beta_{\infty}=\int_{0}^{\frac{1}{2}}\left(1-e^{-t}\right) \frac{d t}{t}-\int_{\frac{1}{2}}^{\infty} e^{-t} \frac{d t}{t},
$$

The assertion (5.31) is a special case of an identity valid for each $w>0$, that

$$
\int_{0}^{w}\left(1-e^{-t}\right) \frac{d t}{t}-\int_{w}^{\infty} e^{-t} \frac{d t}{t}=\gamma+\log w
$$

on taking $w=\frac{1}{2}$. The proof of this identity reduces to the case $w=1$, which easily follows from an integral formula for Euler's constant

$$
\gamma=\int_{0}^{1}\left(1-e^{-t}-e^{-\frac{1}{t}}\right) \frac{d t}{t}
$$

found by Barnes [1].

Remark. Computational evidence of Maslanka (private communication) suggests that $S_{\infty}(n, \pi)$ has a full asymptotic expansion in inverse powers $n^{-k}$ with $k \geq 0$. The further coefficients of such expansions will likely depend on the archimedean parameters $\left\{\kappa_{j}(\pi): 1 \leq j \leq N\right\}$, unlike the first two coefficients appearing in Theorem 5.1 .

\section{Bounds for $S_{f}(n, \pi)$}

We obtain a bound for $S_{f}(n, \pi)$ in terms of the incomplete Li coefficient to height $T$, defined by

$$
\Omega_{n}(T, \pi):=\sum_{\substack{\rho \in Z(\pi) \\|\Im(\rho)| \leq T}} 1-\left(1-\frac{1}{\rho}\right)^{n},
$$

where $T$ is a cutoff parameter.

\footnotetext{
${ }^{4}$ This identity was found by comparison of the author's original formula [5.30) with an asymptotic formula for the Li coefficients (under RH) given by Voros [50, eqn. (11)].
} 
Theorem 6.1. For any irreducible cuspidal (unitary) automorphic representation on $G L(N)$ and any fixed $\beta>\frac{1}{\sqrt{2}}$ there holds

$$
S_{f}(n, \pi)=\Omega_{n}\left(\sqrt{\beta n}, \pi^{\vee}\right)+O(\sqrt{n}),
$$

in which the implied constant in the $O$-notation depends on both $\beta$ and $\pi$. If the Riemann hypothesis holds for $L(s, \pi)$ then

$$
S_{f}(n, \pi)=O(\sqrt{n} \log n) .
$$

Proof. We use a contour integral argument. We introduce the kernel function

$$
k_{n}(s):=\left(1+\frac{1}{s}\right)^{n}-1=\sum_{j=1}^{n}\left(\begin{array}{l}
n \\
j
\end{array}\right)\left(\frac{1}{s}\right)^{j},
$$

If $C_{1}$ is a contour enclosing the point $s=0$ counterclockwise on a circle of small enough positive radius $R$, the residue theorem gives

$$
\frac{1}{2 \pi i} \int_{C_{1}} k_{n}(s)\left(-\frac{L^{\prime}}{L}(s+1, \pi)\right)=\sum_{j=1}^{n}\left(\begin{array}{l}
n \\
j
\end{array}\right) \eta_{j-1}=S_{f}(n, \pi) .
$$

The residue comes entirely from the singularity at $s=0$, as no other singularities lie inside the contour.

We deform the contour to the counterclockwise oriented rectangular contour $C_{2}(n)$ consisting of vertical lines with real part $\Re(s)=\sigma_{0}$ and $\Re(s)=\sigma_{1}$ where we will choose $-3<\sigma_{0}<-2$, and $\sigma_{1}=2 \sqrt{n}$, and horizontal lines at $\Im(s)= \pm T$, where we will choose $T=\sqrt{\beta n}+\epsilon_{n}$, for some $0<\epsilon_{n}<1$. The integrand has simple poles at the zeros of $L(s, \pi)$, and some of them will now lie inside the contour. The residue theorem gives

$$
I_{2}(n):=\int_{C_{2}(n)} k_{n}(s)\left(-\frac{L^{\prime}}{L}(s+1, \pi)\right)=S_{f}(n, \pi)+\sum_{\substack{\rho \in Z(\pi) \\|\Im(\rho)|<T}}\left(1+\frac{1}{\rho-1}\right)^{n}-1+I_{2}(n, \text { triv }),
$$

in which $I_{2}(n$, triv $)$ evaluates the residues coming from the trivial zeros of $L(s, \pi)$ which will satisfy $-3 \leq \Re(s)<\frac{1}{2}$. The trivial zeros are associated to the archimedean factors and their location is dictated by the values $\mu_{j}(\pi)$, which by Theorem 2.1 satisfy $\Re\left(\kappa_{j}(\pi)\right)>-\frac{1}{2}$, so that all trivial zeros satisfy $\Re(\rho)<\frac{1}{2}$. We have

$$
\left|1+\frac{1}{z-1}\right|=\left|\frac{z}{z-1}\right|<1 \quad \text { when } \quad \Re(z)<\frac{1}{2},
$$

and using this we conclude that the residue at each trivial zero is $O(1)$. Since the trivial zeros fall in $N$ arithmetic progressions with spacing 2, there are $O(N)$ such zeros in the interval $-3 \leq \Re(s)<\frac{1}{2}$ we conclude that the trivial zero contribution is $I_{2}(n, \operatorname{triv})=O(1)$, with $O$-constant depending on $\pi$.

Using the symmetry $\rho \mapsto 1-\bar{\rho}$ of $Z(\pi)$, which does not change $|\Im(\rho)|$, we can rewrite the sum over $Z(\pi)$ in terms of

$$
\left(\frac{1-\bar{\rho}}{-\bar{\rho}}\right)^{n}-1=\left(\frac{\bar{\rho}-1}{\bar{\rho}}\right)^{n}-1
$$


We observe that $\bar{\rho} \in Z\left(\pi^{\vee}\right)$ so that (6.5) can be rewritten

$$
I_{2}(n)=S_{f}(n, \pi)-\Omega_{n}\left(T, \pi^{\vee}\right)+O(1) .
$$

The main part of the theorem will be to establish

$$
I_{2}(n)=O(\sqrt{n} \log n) .
$$

The theorem will follow from the two assertions that $I_{2}(n)=O\left(\frac{n}{\log n}\right)$ and

$$
\left|\Omega_{n}\left(\beta \frac{n}{\log n}, \pi^{\vee}\right)-\Omega_{n}\left(T, \pi^{\vee}\right)\right|=O(\sqrt{n} \log n) .
$$

The second assertion follows from the observation that $\left|T-\beta \frac{n}{\log n}\right|<1$, that there are $O(\log n)$ zeros in an interval of length one at this height (by (2.11)), and that for each zero $\rho=\beta+i \gamma$ $\beta \frac{n}{\log n} \leq|\Im(\rho)|<\beta \frac{n}{\log n}+1$ there holds

$$
\left|\left(\frac{\rho-1}{\rho}\right)^{n}\right| \leq\left|\frac{1+i T}{i T}\right|^{n} \leq\left|1+\frac{\log n}{\beta n}\right|^{n} \leq \exp (\beta \log n)<n^{\frac{1}{2}} .
$$

It remains to bound $I_{2}(n)$. We will use the following estimate for $\frac{L^{\prime}}{L}(s, \pi)$ in a region including the critical strip.

Lemma 6.1. For $-4 \leq \Re(s)<4$ and $s=\sigma+i$ there holds

$$
\frac{L^{\prime}}{L}(s, \pi)=\sum_{\{\rho:|\Im(\rho-s)|<1\}} \frac{1}{s-\rho}+O\left(\log \mathfrak{q}(\pi, s) \frac{L^{\prime}}{L}(2, \pi)\right),
$$

in which $\mathfrak{q}(s, \pi)=Q(\pi) \prod_{i=1}^{N}\left(\left|s+\kappa_{j}(\pi)\right|+3\right)$ is the analytic conductor of $\pi$, and the constant in the $O$-notation is absolute.

Proof. The classical proof for Dirichlet $L$-functions [12, Chap. 15, p. 102] generalizes to give (6.8), following Chapter 5 of [28. If we know the Ramanujan conjecture holds for $L(s, \pi)$, then we can control the size of $\frac{L^{\prime}}{L}(2, \pi)$ in the remainder term.

We now choose the parameters $\sigma_{0}$ and $T$ appropriately to avoid poles of the integrand. Since the trivial zeros of $L(s, \pi)$ fall in $N$ arithmetic progressions with spacing 2, we may choose $\sigma_{0}$ so that the contour avoids coming within $\frac{1}{2 N}$ of any trivial zero. Similarly we can choose $T=\beta \frac{n}{\log n}+\epsilon_{n}$ with $0 \leq \epsilon_{n} \leq 1$ so that the horizontal lines do not approach closer than $O(\log n)$ to any zero of $L(s, \pi)$. It then follows from Lemma 6.1 that on the horizontal line in the interval $-3 \leq \Re(s) \leq 4$ we have

$$
\left|\frac{L^{\prime}}{L}(s+1, \pi)\right|=O\left((\log T)^{2}\right) .
$$

The Euler product for $L(s, \pi)$ converges absolutely for $\Re(s)>1$ (by Theorem 2.1(1)) hence the Dirichlet series for $\frac{L^{\prime}}{L}(s, \pi)$ converges absolutely for $\Re(s)>1$. More precisely if $\frac{L^{\prime}}{L}(s, \pi)=\sum_{m=2}^{\infty} b(m, \pi) m^{-s}$ then for $\sigma>1$,

$$
\left|\frac{L^{\prime}}{L}\right|(\sigma)=\sum_{m=2}^{\infty}|b(m, \pi)| m^{-\sigma}<\infty .
$$


Since the Dirichlet series for $\frac{L^{\prime}}{L}(s, \pi)$ has no constant term, we obtain for $\Re(s)=\sigma>2$ the bound

$$
\left|\frac{L^{\prime}}{L}(s, \pi)\right| \leq\left|\frac{L^{\prime}}{L}\right|(\sigma, \pi)\left|\leq 2^{-(\sigma-2)}\right| \frac{L^{\prime}}{L}(2, \pi) \mid .
$$

We now consider the integral $I_{2}(n)$ on the vertical segment (I) having $\Re(s)=\sigma_{1}$, call it $I_{2}^{(I)}(n)$. We have

$$
\left|\left(1-\frac{1}{s}\right)^{n}-1\right| \leq\left(1+\frac{1}{\sigma_{1}}\right)^{n}+1 \leq\left(1+\frac{1}{2 \sqrt{n}}\right)^{n} \leq \exp \left(\frac{1}{2} \sqrt{n}\right)<2^{\sqrt{n}} .
$$

Now the estimate (6.10) gives on (I) that

$$
\left|\frac{L^{\prime}}{L}(s, \pi)\right| \leq C_{0} 2^{-2(\sqrt{n}+2)} .
$$

so that the integrand is bounded above by $C_{1} 2^{-\sqrt{n}}$. The length of the contour is $O\left(\frac{n}{\log n}\right)$ so we obtain the estimate $\left|I_{2}^{(I)}(n)\right|=o(1)$.

We next consider the integral $I_{2}(n)$ on the two horizontal segments, call them (II) and (IV). It suffices to bound $I_{2}^{(I I)}(n)$, the treatment for (IV) being identical. Let $s=\sigma+i t$ be a point on (II). We have $T \geq \sqrt{\beta n}$ so that

$$
\begin{aligned}
\left|1+\frac{1}{s}\right| & \left.=\mid\left(1+\frac{\sigma}{\sigma^{2}+T^{2}}\right)+\frac{i T}{\sigma^{2}+T^{2}}\right) \mid \\
& \leq\left(\left(1+\frac{\sigma}{\sigma^{2}+T^{2}}\right)^{2}++\frac{1}{\sigma^{2}+T^{2}}\right)^{\frac{1}{2}} \\
& \leq 1+\frac{\sigma+1}{\sigma^{2}+T^{2}} .
\end{aligned}
$$

By hypothesis $T^{2} \geq \beta n$ so for $-3 \leq \sigma \leq 3$ we have

$$
\left|k_{n}(s)\right| \leq\left(1+\frac{4}{16+\beta n}\right)^{n}+1 \leq \exp \left(\frac{4}{\beta}\right)+1=O(1)
$$

On this interval Lemma 6.1 gives

$$
\left|\frac{L^{\prime}}{L}(s, \pi)\right|=O\left((\log T)^{2}\right)=O\left((\log n)^{2}\right)
$$

since we have chosen the ordinate $T$ to stay away from zeros of $L(s, \pi)$. Now we step across the interval (II) towards the right, in segments of length 1 , starting from $\sigma=3$. At the initial point we have $\left|\frac{L^{\prime}}{L}(s, \pi)\right|=O(1)$ since we are in the absolute convergence region, and the estimate (6.10) gains a factor of 2 going one unit to the right. In comparison

$$
\begin{aligned}
\left|\frac{k_{n}(s+1)+1}{k_{n}(s)+1}\right| & =\left|\frac{1+\frac{1}{\sigma+1+i T}}{1+\frac{1}{\sigma+i T}}\right|^{n} \\
& \leq \mid 1+\left(\frac{1}{\sigma+1+i T}-\left.\frac{1}{\sigma+i T}\right|^{n}\right. \\
& \leq\left(1+\frac{1}{|\sigma+1+i T||\sigma+i T|}\right)^{n} \\
& \leq\left(1+\frac{1}{T^{2}}\right)^{n} \leq e^{\beta}<2 .
\end{aligned}
$$


We obtain an upper bound for $\mid k_{n}(s) \frac{L^{\prime}}{L}(s, \pi)$ that decreases geometrically at each step, and after $O(\log n)$ steps it becomes $O(1)$ so we obtain the upper bound

$$
\left|I_{2}^{(I I)}(n)\right|=O\left(\sqrt{n}+(\log n)^{3}\right)=O(\sqrt{n}) .
$$

A similar bound holds for $\left|I_{2}^{(I V)}(n)\right|$.

For the remaining vertical segment (III) with $\Re(s)=\sigma_{0}$, we have that the kernel function $\left|k_{n}(s)\right|=O(1)$ on the line segment (III) by (6.6), and the $\mid \frac{l^{\prime}}{L}(s, \pi)=O(\log (|s|+2))$ using Lemma 6.1 Since the segment (III) has length $O(\sqrt{n})$ we obtain the bound

$$
\left|I_{2}^{(I I I)}(n)\right|=O(\sqrt{n} \log n) .
$$

Using this bound will already suffice to get (6.2) with the remainder term $O(\sqrt{n} \log n)$.

We can save an extra logarithm in this estimate, to improve (6.13) to $O(\sqrt{n})$, as follows. We apply the functional equation to move the $L$-function to the right half plane $\Re(s)>2$. The functional equation gives

$$
\begin{aligned}
\frac{L^{\prime}}{L}(s, \pi) & =\frac{L^{\prime}}{L}\left(1-s, \pi^{\vee}\right)+\sum_{j=1}^{N}\left(\frac{\Gamma_{\mathbb{R}}^{\prime}}{\Gamma_{\mathbb{R}}}\left(s+\kappa_{j}\right)+\frac{\Gamma_{\mathbb{R}}^{\prime}}{\Gamma_{\mathbb{R}}}\left(1-s+\kappa_{j}\right)\right)+C_{1}(\pi) \\
& =\frac{L^{\prime}}{L}\left(1-s, \pi^{\vee}\right)+\frac{1}{2} \sum_{j=1}^{N}\left(\frac{\Gamma^{\prime}}{\Gamma}\left(\frac{s+\kappa_{j}}{2}\right)+\frac{\Gamma^{\prime}}{\Gamma}\left(\frac{1-s+\kappa_{j}}{2}\right)\right)+C_{2}(\pi) .
\end{aligned}
$$

Thus the estimate of $I_{2}^{(I I I)}(n)$ divides into two terms, an $L$-function term and an archimedean term. For the $L$-function term, the line $\Re(s)=\sigma_{0}$ is moved to $\Re s=1-\sigma_{0}>3$, so we are in the absolute convergence region with

$$
\left|\frac{L^{\prime}}{L}\left(1-s, \pi^{\vee}\right)\right|=O(1) .
$$

and this inequality saves one logarithm in the upper bound. For the archimedean terms we save a logarithm as follows. The fact that the quantities $\kappa_{j}(\pi)$ are permuted under complex conjugation (Theorem $\mathbf{3 . 1}(2)$ ), that $k_{n}(s)$ is real on the real axis, and that the segment (III) is invariant under complex conjugation ensures that the contribution to $I_{2}^{(I I I)}(n)$ coming from the archimedean terms is invariant under complex conjugation. It therefore suffices to bound the real part of the relevant integrals. We have two such integrals

$$
J_{1}:=\int_{(I I I)} \frac{\Gamma^{\prime}}{\Gamma}\left(\frac{\sigma_{0}+i t+1+\kappa_{j}(\pi)}{2}\right) d t .
$$

and

$$
J_{2}:=\int_{(I I I)}\left(1+\frac{1}{s}\right)^{n} \frac{\Gamma^{\prime}}{\Gamma}\left(\frac{\sigma_{0}+i t+1+\kappa_{j}(\pi)}{2}\right) d t
$$

The idea can be seen most clearly in the first integral. We have, using Stirling's formula,

$$
\begin{aligned}
\Re\left(J_{1}\right)= & \Re\left(\left[\left.2 \log \Gamma\left(\left(\frac{\sigma_{0}+i t+1+\kappa_{j}}{2}\right)\right]\right|_{-T} ^{T}\right)\right. \\
= & 2 \Re((\sigma+i T)(\log |\sigma+i T|+i \arg (\sigma+i T)) \\
& \quad-(\sigma-i T)(\log |\sigma-i T|-i \arg (\sigma-i T)))+O(T) \\
= & -2 \pi T+O(T)=O(\sqrt{n}) .
\end{aligned}
$$


in which the $2 \pi T$ in the last term comes from $\arg (\sigma+i T) \approx \frac{\pi}{2}$ and $\arg (\sigma-i T) \approx-\frac{\pi}{2}$. (In contrast to (6.15) we have $\left|\Im\left(J_{1}\right)\right|>c T \log T$ and no savings is possible.) In the second integral one can show that

$$
\left|J_{2}\right|=O(T)=O(\sqrt{n})
$$

To see this, set

$$
1+\frac{1}{\sigma_{0}+i t}=R e^{i \phi(t)}
$$

The idea is that $R=1-\frac{1}{t^{2}}+O\left(\frac{1}{t^{3}}\right)$ and $\phi(t)=\frac{1}{t}+O\left(\frac{1}{t^{2}}\right)$. Then $\left(1+\frac{1}{\sigma_{0}+i t}\right)^{n}=R^{n} e^{i n \phi t}$ rotates though about $C \log T$ cycles as the integrand varies from $-T$ to $T$. The integrand changes nonlinearly, but remains approximately constant over each cycle, changing by a factor of $1+O\left(\frac{1}{\log T}\right)$ over the range $\frac{T}{\log T} \leq|t| \leq T$. The net effect of the averaging over the unit circle is to save one factor of $\log T$, in both the real and the imaginary part. Combining these bounds gives

$$
\left|I_{2}^{(I I I)}(n)\right|=O(\sqrt{n}) .
$$

Totalling all these bounds above gives

$$
S_{f}(n, \pi)=\Omega_{n}\left(T, \pi^{\vee}\right)+O(\sqrt{n}),
$$

with $T=\sqrt{\beta n}+\epsilon_{n}$. To establish (6.2) it remains to show that changing the value of $T$ in the incomplete Li coefficient by at most 1 does not alter the asymptotics. We show that

$$
\left|\Omega_{n}\left(\sqrt{\beta n}, \pi^{\vee}\right)-\Omega_{n}\left(T, \pi^{\vee}\right)\right|=O(\sqrt{n} \log n) .
$$

This follows from the observation that there are $O(\log n)$ zeros in an interval of length one at this height (by (2.11) ), and that for each zero $\rho=\beta+i \gamma$ with $\beta \frac{n}{\log n} \leq|\Im(\rho)|<\beta \frac{n}{\log n}+1$ there holds

$$
\left|\left(\frac{\rho-1}{\rho}\right)^{n}\right| \leq\left|\frac{1+i T}{i T}\right|^{n} \leq\left|1+\frac{\log n}{\beta n}\right|^{n} \leq \exp (\beta \log n)<\sqrt{n} .
$$

This establishes (6.2).

Now suppose that the Riemann hypothesis holds for $\xi(s, \pi)$. Then we have

$$
\left|1-\frac{1}{\rho-1}\right|=\left|\frac{\rho}{\rho-1}\right|=\left|\frac{-\frac{1}{2}+i \gamma}{\frac{1}{2}+i \gamma}\right|=1
$$

It follows that each zero contributes a term of absolute value at most 2 to the incomplete $\mathrm{Li}$ coefficient $\Omega_{n}(T, \pi)$, and we obtain

$$
\Omega_{n}(\sqrt{\beta n}, \pi)=O(\sqrt{n} \log n),
$$

on using the zero density estimate in Theorem 2.17(4). Here the constant in the $O$-notation depends on $\beta$ and $\pi$.

\section{Interpolation Function for Li Coefficients}

We construct an entire function that interpolates the Li coefficients at integer values. 
Theorem 7.1. (1) For any irreducible cuspidal unitary automorphic representation $\pi$ over $G L(N)$ over $\mathbb{Q}$, there exists an entire function $F_{\pi}(z)$ of order one and exponential type having the two properties:

(i). It interpolates the generalized Li coefficients $\lambda_{n}(\pi)$ at integer values, i.e.

$$
F_{\pi}(n)=\lambda_{n}(\pi), \quad n \in \mathbb{Z} .
$$

(ii) It is real-valued on the imaginary axis, with

$$
F_{\pi}(-\bar{z})=\overline{F_{\pi}(z)} .
$$

(2) If the Riemann hypothesis holds for $L(s, \pi)$, then there exists a unique function $F_{\pi}(z)$ of exponential type at most $\pi$ that satisfies (i), and has the property that if $\xi(s, \pi)$ has a $k$-th order zero at $s=\frac{1}{2}$, then $F_{\pi}^{*}(z)=F_{\pi}(z)-k(1-\cos \pi z)$ is of exponential type strictly less than $\pi$. On the real axis, this function satisfies the bound

$$
\left|F_{\pi}(x)\right| \leq C(|x|+2) \log (|x|+2),
$$

for some constant $C$ depending on $\pi$.

Proof. In this proof we regard $\pi$ as fixed, with zeros $\rho$ drawn from the multiset $Z(\pi)$.

(1). We define

$$
\left(1-\frac{1}{\rho}\right)^{z}:=\exp \left(z \log \left(1-\frac{1}{\rho}\right)\right)
$$

in which we take the principal branch of the logarithm, with argument $-\frac{\pi}{2}<\Im(\log z) \leq \frac{\pi}{2}$.

We also have the binomial expansion

$$
\left(1-\frac{1}{\rho}\right)^{z}=\sum_{j=0}^{\infty}(-1)^{j}\left(\begin{array}{l}
z \\
j
\end{array}\right) \frac{1}{\rho^{j}} .
$$

When $|\rho|>1$ this expansion converges absolutely for all $z \in \mathbb{C}$, since the terms in the series eventually decay geometrically in absolute value.

We now let $R>0$ and define

$$
F_{R}(z)=\sum_{|\rho| \leq R}\left(1-\left(1-\frac{1}{\rho}\right)^{z}\right)+(-z)\left(\sum_{\{|\rho|>R}{ }^{\prime} \frac{1}{\rho}\right)+\sum_{j=2}^{\infty}(-1)^{j}\left(\begin{array}{l}
z \\
j
\end{array}\right)\left(\sum_{|\rho|>R} \frac{1}{\rho^{j}}\right)
$$

Here

$$
\sum_{|\rho|>R}{ }^{\prime} \frac{1}{\rho}:=\lim _{T \rightarrow \infty} \sum_{R<|\rho| \leq T} \frac{1}{\rho}
$$

and this sum is *-convergent by Lemma 2.1. For notational convenience we define the partial power sums

$$
\sigma_{j}(R, \pi):=\sum_{|\rho|>R} \sum \frac{1}{\rho^{j}} .
$$

These sums converge absolutely for $j \geq 2$ and are $*$-convergent for $j=1$, by Lemma 2.1 We can rewrite

$$
F_{R}(z)=\sum_{|\rho| \leq R}\left(1-\frac{1}{\rho}\right)^{z}-\sigma_{1}(R, \pi) z+\sum_{j=2}^{\infty}(-1)^{j}\left(\begin{array}{l}
z \\
j
\end{array}\right) \sigma_{j}(R, \pi)
$$


The zero-counting estimate in Theorem 2.1 (4) imply there are at most $O(\log T)$ zeros in an interval of length 1 at height $T$, where the $O$-constant depends on the representation $\pi$. Therefore we obtain, for $j \geq 2$

$$
\begin{aligned}
\left|\sigma_{j}(R, \pi)\right| & \leq \sum_{m=R}^{\infty} \sharp\{m \leq|\rho|<m+1\} \frac{1}{m^{j}} \\
& \leq \sum_{m=R}^{\infty} C \frac{\log m}{m^{j}}=O\left((\log R)^{2} \frac{1}{R^{j-1}}\right) .
\end{aligned}
$$

where the $O$-constant depends on $\pi$. For the term $j=1$ we similarly obtain a $*$-convergence estimate

$$
\left|\sigma_{1}(R, \pi)\right|=O\left(\frac{(\log R)^{2}}{R}\right)
$$

by partial summation, cancelling the zeros at height $T$ against those at height $-T$. We therefore have, on the disk $|z| \leq \sqrt{R}$, that

$$
\begin{aligned}
\left|\left(\begin{array}{c}
z \\
j
\end{array}\right) \sigma_{j}(R, \pi)\right| & \leq\left|\left(\begin{array}{l}
z \\
j
\end{array}\right)\right|\left|\sigma_{j}(R, \pi)\right| \\
& \leq C_{1}\left(\prod_{k=1}^{j} \frac{\sqrt{R}+k}{k}\right)(\log R)^{2} R^{-j+1} \\
& \leq C_{1}\left(\prod_{k=1}^{j} \frac{1+\frac{k}{\sqrt{R}}}{k}\right)(\log R)^{2} R^{-\frac{j}{2}+1} \\
& \leq C_{2}(\log R)^{2} R^{-\frac{j}{2}+1}
\end{aligned}
$$

On summing over $j \geq 1$ it follows that the terms on the right side of (7.9) converges uniformly on the entire disk $|z|<\sqrt{R}$ to an analytic function $F_{R}(z)$.

We also obtain from this a bound on the maximum modulus of $F_{R}(z)$ on $|z| \leq R$. Set

$$
\beta(\pi):=\sup _{\rho \in Z(\pi)}\left|\log \left(1-\frac{1}{\rho}\right)\right|,
$$

and observe $\beta(\pi)$ is finite because $1 \notin Z(\pi)$, and $\left.\mid 1-\frac{1}{\rho}\right) \mid \rightarrow 0$ as $|\rho| \rightarrow \infty$. then we obtain, for $|z|=\leq \sqrt{R} \geq 2$, that

$$
\begin{aligned}
\left|F_{R}(z)\right| & \leq \sum_{|\rho| \leq R} e^{\sqrt{R} \log \left(1-\frac{1}{\rho}\right)}+O\left((\log R)^{2} \frac{1}{1-R^{-\frac{1}{2}}}\right) \\
& =C_{3} R \log \left(R e^{\beta(\pi) \sqrt{R}}\right)
\end{aligned}
$$

in which the $O$-constant depends on $\pi$.

We assert that all $F_{R}(z)$ represent the same analytic function. If $R_{1}<R_{2}$ and we consider the functions on the domain $|z|<\sqrt{R_{1}}$ we can expand the individual terms $\left(1-\frac{1}{\rho}\right)^{z}$ with $R_{1}<|\rho| \leq R_{2}$ in $F_{R_{2}}(z)$ and combine them term-by-term with the binomial expression, using

$$
\sigma_{j}\left(R_{1}, \pi\right)=\sigma_{j}\left(R_{2}, \pi\right)+\sum_{R_{1}<|\rho| \leq R_{2}} \frac{1}{\rho_{j}}
$$


Since all sums converge absolutely and uniformly on $|z|<\sqrt{R_{1}}$, we conclude that $F_{R_{2}}(z)$ agrees with $F_{R_{1}}(z)$ there. Letting $R \rightarrow \infty$, we obtain an entire function $F_{\pi}(z)$ such that

$$
F_{\pi}(z)=F_{R}(z) \quad \text { when } \quad|z| \leq \sqrt{R}
$$

The maximum modulus bound (17.13) now establishes that $F_{\pi}(z)$ is an entire function of order one and exponential type at most $\beta(\pi)$.

Evaluating at integer points $n \in \mathbb{Z}$ and choosing $R=2 n^{2}$ gives

$$
\begin{aligned}
F_{\pi}(n) & =\sum_{|\rho| \leq R}\left(1-\left(1-\frac{1}{\rho}\right)^{n}\right)-n \sigma_{1}(R, \pi)+\sum_{j=2}^{\infty}(-1)^{j}\left(\begin{array}{l}
n \\
j
\end{array}\right) \sigma_{j}(R, \pi) \\
& =\sum_{|\rho| \leq R}\left(1-\left(1-\frac{1}{\rho}\right)^{n}\right)-n \sigma_{1}(R, \pi)+\sum_{j=2}^{n}(-1)^{j}\left(\begin{array}{l}
n \\
j
\end{array}\right) \sigma_{j}(R, \pi) .
\end{aligned}
$$

The last term is a finite sum, so we can expand all the terms in the first sum $|\rho|<R$ and rearrange to obtain

$$
F_{\pi}(n)=-n \sigma_{1}(\pi)+\sum_{j=2}^{n}(-1)^{j}\left(\begin{array}{l}
n \\
j
\end{array}\right) \sigma_{j}(\pi)=\lambda_{n}(\pi)
$$

using Lemma 2.2 for the last equality. This proves property (i).

The symmetry property of zeros under $\rho \mapsto 1-\bar{\rho}$ implies that $F_{R}(z)$ is real on the imaginary axis, for $|z|<R$. It follows that $F_{\pi}(z)$ is real on the imaginary axis, and reflection principle then gives the symmetry

$$
F_{\pi}(-x+i y)=\overline{F_{\pi}(x+i y)} \text { when }|z|<R .
$$

which is property (ii).

We have constructed a specific function $F_{\pi}(z)$ which has properties (i), (ii). If $\beta(\pi) \geq \pi$, then properties (i), (ii) do not determine the function uniquely because we can add a real multiple of of $z \sin \pi z$ to the function while preserving properties (i) and (ii).

(2) Now suppose that the Riemann hypothesis holds for $\xi(s, \pi)$. Then we can write

$$
\rho=\frac{1}{2}+i \gamma=|\rho| e^{i \varphi_{\rho}}
$$

with $-\pi<\phi_{\rho} \leq \pi$, and

$$
\varphi_{\rho}:=\tan ^{-1}(2 \gamma),
$$

so that $\phi_{\rho} \geq 0$ when $\gamma \geq 0$, and $\varphi_{\rho}<0$ otherwise. We have

$$
1-\frac{1}{\rho}=-\frac{\frac{1}{2}-i \gamma}{\frac{1}{2}+i \gamma}=e^{i\left(-2 \varphi_{\rho} \pm \pi\right)}
$$

with the sign \pm chosen so that the angle falls in $(-\pi, \pi]$. Let us now set

$$
\varphi_{*}(\rho):=\left\{\begin{aligned}
-2 \varphi_{\rho}+\pi & \text { if } \varphi_{\rho}>0 \leftrightarrow \gamma>0 \\
-2 \varphi_{\rho}-\pi & \text { if } \varphi_{\rho}<0 \leftrightarrow \gamma<0
\end{aligned}\right.
$$


The case $\varphi_{\rho}=0$, corresponding to $s=\frac{1}{2}$, is problematic, since the sign $\pm \pi$ must have a discontinuity there. For the moment we use the convention that the + sign is chosen. Consequently

$$
\left|\log \left(1-\frac{1}{\rho}\right)\right|=\left|i \varphi^{*}(\rho)\right| \leq \pi
$$

and equality can occur only if $\varphi^{*}(\rho)=\pi$, which requires that $\varphi_{\rho}=0$ and $\rho=\frac{1}{2}$. It follows that if the Riemann hypothesis holds for $Z(\pi)$ then $\beta(\pi) \leq \pi$, and if there is no zero at $s=\frac{1}{2}$ then $\beta(\pi)<\pi$.

For uniqueness, suppose first that $Z(\pi)$ includes no zero at $s=\frac{1}{2}$. It is well known (from the sampling theorem) that an entire function of order one and exponential type less than $\pi$ is completely specified by its values at integer points. Indeed, the difference of two such functions would be an entire function of order one and exponential type $\tau$ less than $\pi$ vanishing at all integer points. Since a function of exponential type $\tau$ has at most $(\tau+o(1)) R$ zeros in a disk of radius $R$, as $R \rightarrow \infty$, it must be identically zero. In this case property (i) together with a growth bound on the function characterizes the interpolation function uniquely. In the cases where $Z(\pi)$ has zeros at $s=\frac{1}{2}$, we use a convention to handle their contribution and define $F_{\pi}(z)$ uniquely. If it has exactly $k$ zeros there, we define their contribution to be

$$
f_{\frac{1}{2}}(z):=\frac{k}{2}\left(e^{-i \pi z}+e^{i \pi z}\right)
$$

This function is real on the imaginary axis, and has $f_{\frac{1}{2}}(z)=f_{\frac{1}{2}}(-z)$, and it corresponds to half of the zeros at $s=\frac{1}{2}$ are assigned argument $-\pi i$ and half are assigned argument $\pi i$. Subtracting this contribution leaves an interpolation function of exponential type strictly less than $\pi$, which is then uniquely specified by condition (i).

It remains to bound the size of the interpolation function $F_{\pi}(z)$ on the real axis. Under the Riemann hypothesis, the bounds on the Li coefficients in $\S 5$ and $\S 6$ lead one to expect an upper bound of shape $O((|x|+2) \log (|x|+2))$, and we now show this holds. We have

$$
F_{\pi}(x)=\sum_{\rho}\left(1-\left(1-\frac{1}{\rho}\right)^{x}\right)
$$

and we bound each term separately in absolute value. For a zero $\rho$ with $\gamma>0$ we have

$$
\varphi_{\rho}=\frac{\pi}{2}-\frac{1}{2 \gamma}+O\left(\frac{1}{\gamma^{2}}\right)
$$

It follows that for $\gamma>5|x|$ we have

$$
\begin{aligned}
1-\left(1-\frac{1}{\rho}\right)^{x} & =1-e^{i x\left(-2 \varphi_{\rho}+\pi\right)} \\
& =1-\exp \left(2 i x\left(\frac{1}{2 \gamma}+O\left(\frac{1}{\gamma^{2}}\right)\right)\right. \\
& =i \frac{i x}{\gamma}+O\left(\frac{x}{\gamma^{2}}\right) .
\end{aligned}
$$

We find a similar expression when $\gamma<0$.

We now divide the sum (7.23) into parts $|\rho|<|x|+2$ and $|\rho| \geq|x|+2$. The former sum contributes $O((|x|+2) \log (|x|+2))$ using the bounds on the number of zeros in Theorem $2.1(4)$. 
On the remaining range we pair the zeros with increasing positive $\gamma$ against those with increasing negative $\gamma$, and find that their imaginary parts cancel out by Theorem [2.1(4) to an error $O\left(\frac{x \log (|\gamma|+2)}{\gamma^{2}}\right)$ on each zero. Now summing over the $O(\log T)$ zeros in an interval of length one at height $T$, for $|x|+2 \leq T \leq \infty$ gives a total contribution of

$$
O\left(x(\log |x|+2)^{2} \frac{1}{|x|+2}\right)=O\left((\log |x|+2)^{2}\right) .
$$

The bound on the sum (17.23) is then

$$
\left|F_{\pi}(x)\right| \leq C(|x|+2) \log (|x|+2), \quad-\infty<x<\infty,
$$

as asserted.

We next consider the Fourier transform of $F_{\pi}(x)$ on the real line, regarded as a distribution. We suppose the Riemann hypothesis holds for $\pi$, and consider the unique function $F_{\pi}(z)$ given in Theorem 17.1(2). The growth bound in (7.3) implies that it is well-defined as a tempered distribution. Viewing (7.23) term-by-term we obtain, a representation for this Fourier transform formally as a sum of delta functions

$$
\hat{F}_{\pi}(\eta)=\sum_{\rho \in Z(\pi)}\left(\delta_{0}(\eta)-\delta_{\varphi^{*}(\rho)}(\eta)\right)
$$

in which $\delta_{\alpha}(\eta)$ denotes a delta function centered at $\eta=\alpha$. In viewing this as defining a continuous linear functional on test function in the Schwartz space $\mathcal{S}(\mathbb{R})$, the individual terms in the sum must be grouped as indicated. One deduces that the support of this tempered distribution is real and lies in $[-\pi, \pi]$, and is a discrete set, determined by the delta functions, except at the non-isolated limit point $\eta=0$.

Remarks. (1) One can carry out a similar procedure to interpolate the archimedean contributions $S_{\infty}(n, \pi)$, for $n \geq 1$ with an interpolation function $F_{\pi, \infty}(z)$ that is an entire function of exponential type. One must modify the proof to subtract off the contribution of the term linear in $z$ from the other terms in the formula of Lemma 4.2. to get a convergent formula. The resulting interpolation function is, formally,

$$
F_{\pi, \infty}(z)=C_{3}(\pi)+C_{4}(\pi) z+\sum_{j=1}^{N} \sum_{n=1}^{\infty}\left(1-\frac{2}{2 n+\kappa_{j}(\pi)-1} z-\left(1-\frac{2}{2 n+\kappa_{j}(\pi)-1}\right)^{z}\right)
$$

for a certain constants $C_{3}(\pi), C_{4}(\pi)$. Here we have

$$
\left|1-\frac{2}{2 n+\kappa_{j}(\pi)-1}\right|<1 \text { for all } n \geq 1
$$

The interpolation function $F_{\pi, \infty}(z)$ will be an entire function of exponential type. It has polynomial growth on the positive real axis (bounded by $O((|x|+2) \log (|x|+2)$ for positive real $x)$, but increases exponentially on the negative real axis. It consequence its Fourier transform must be interpreted as a distribution and not as a tempered distribution.

(2) One can also obtain an entire function $F \pi, f(z)$ of exponential type interpolating $S_{f}(n, \pi)$, given as

$$
F_{\pi, f}(z):=F_{\pi}(z)-F_{\pi, \infty}(z)-\delta(\pi)
$$


Again, even assuming the Riemann hypothesis, its Fourier transform must be interpreted as a distribution and not a tempered distribution.

(3) Assume the Riemann hypothesis holds for $L(s, \pi)$, and suppose that $\pi=\pi^{\vee}$ is self-dual. Then (7.20) yields

$$
\left(1-\left(1-\frac{1}{\rho}\right)^{n}\right)+\left(1-\left(1-\frac{1}{1-\rho}\right)^{n}\right)=2-2 \cos n \varphi^{*}(\rho) .
$$

Note that $\varphi_{\rho} \rightarrow \pm \frac{\pi}{2}$ as $\gamma \rightarrow \pm \infty$, and the $\pm \pi$ term is chosen of opposite sign, whence $-2 \varphi_{\rho} \pm \pi \rightarrow 0$ as $|\rho| \rightarrow \infty$.

\section{Concluding Remarks}

(1) Instead of the $\xi$-function treated in $\S 2$, one may alternatively consider generalized Li coefficients associated to the function $\xi^{+}(s, \pi)$ obtained by removing any zeros at $s=\frac{1}{2}$ from $\xi(s, \pi)$, Here

$$
\xi^{+}(s, \pi):=\left(s-\frac{1}{2}\right)^{-e\left(\frac{1}{2}, \pi\right)} \xi(s, \pi),
$$

and we let $\lambda_{n}^{+}(\pi)$ denote the associated Li coefficients. Use of this function has three positive features. First, it allows us to unambiguously obtain the functional equation in the form

$$
\xi^{+}(s, \pi)=\xi^{+}(1-s, \pi)
$$

with the sign convention that $\xi^{+}\left(\frac{1}{2}\right)>0$. Second, the interpolating function $F_{\pi}^{+}(z)$ for the generalized $\mathrm{Li}$ coefficients associated to $\xi^{+}(s, \pi)$ in $\S 7$ will be an entire function of order 1 and type strictly less than $\pi$. In consequence it is uniquely determined by the values $\left\{F_{\pi}^{+}(n, \pi):=\lambda_{n}^{+}(\pi), \quad n \in \mathbb{Z}\right\}$. Third, in terms of the Weil scalar product associated to a hypothetical Hilbert-Polya operator, with eigenvalues $\lambda=s^{2}-\frac{1}{4}$, removal of the zeros at $s=\frac{1}{2}$ would correspond to taking the orthogonal complement of the eigenspace with eigenvalue $\lambda=0$. It is generally believed that zeros at $s=\frac{1}{2}$ should have some arithmetic-geometric meaning, as in the Birch-Swinnerton Dyer conjecture, and there may well be some arithmetico-geometric way to directly characterize this eigenspace.

(2) There are analogues of the Li coefficients for automorphic $L$-functions in the function field case. We first note that the $\xi$-function for the trivial representation over a function field $K$ in one variable over a finite field $\mathbb{F}_{q}$ can be taken to be

$$
\xi\left(s, \pi_{t r i v, K}\right)=q^{-s}\left(1-q^{-s}\right)\left(1-q^{1-s}\right) Z_{K}(s),
$$

in which $Z_{K}(s)$ is the (completed) function field zeta function, and is a polynomial in $w=q^{-s}$. For a rational function field $K=\mathbb{F}_{q}(T)$ we have $\xi\left(s, \pi_{\text {triv }, K}\right)=1$. All other automorphic $L$-functions are polynomials in the variable $w$. The transformation $s=-\frac{z}{1-z}$ is still used to define the Li coefficients in the function field case. The definition (3.1) for the "Weil scalar product" applies, and the positivity of the Li coefficients is interpretable in terms of this scalar product applied to the Li test functions $G_{n}(s)$. The asymptotics of the Li coefficients in the function field case will be quite different from those in the number field case, since there are no archimedean places.

To interpret the Li coefficients in terms of a function field "explicit formula," one must use a version in terms of the $s$-variable, noting that $L$-functions are singly periodic with period 
$\frac{2 \pi i}{\log q}$. The standard function field "explicit formula" is generally stated in terms of the variable $w$, and it has an algebraic geometry interpretation, related to intersection theory. The allowed test functions in this formula (related to divisors) are Laurent polynomials in $w=q^{-s}$ with integer coefficients, cf. Haran [25]. The Li test functions $G_{n}(s)$ are not functions of $q^{-s}$, and must be $q$-periodized using

$$
P_{q}\left(G_{n}\right)(s):=\sum_{n \in \mathbb{Z}} G_{n}\left(s+\frac{2 \pi i n}{\log q}\right)
$$

to be viewed as function field test functions. This sum (8.1) is conditionally convergent and is regularized as a limit as $T \rightarrow \infty$ of $\sum_{-T<n<T}$. It would be interesting to see if this framework is compatible with a framework of Deninger [13, 15, Sect. 4]. Another feature of the function field case is that some function field $L$-functions have multiple zeros on the critical line at positions other than $s \equiv \frac{1}{2}\left(\bmod \frac{2 \pi i}{\log q}\right)$.

(3) Under the Riemann hypothesis, the "explicit formula" decomposition of the Li coefficients into $S_{\infty}(n, \pi)$ and $S_{f}(n, \pi)$ reveals that the dominant contribution to the asymptotics of the $\mathrm{Li}$ coefficients comes from the archimedean terms, which correspond in (7.25) to the "trivial zeros" of $L(s, \pi)$. This fact contrasts with the definition (1.1) of the Li coefficients (1.1) which is a sum over the non-trivial zeros of $L(s, \pi)$, and excludes the "trivial zeros". In effect certain asymptotics of the nontrivial zeros are described in terms of the "trivial zeros." 


\section{Appendix: "Explicit Formula" and Weil's Quadratic Functional}

For simplicity we treat here only the case of the trivial representation $\pi_{\text {triv }}$ on $G L(1)$. In that case, there are two versions of the "explicit formula" of prime number theory, the trace form and the covariance form, described below. For all other automorphic representations $\pi$ there is essentially only one form of the "explicit formula."

Consider first a vector space $\mathcal{A}=\mathcal{A}_{\delta}$ of test functions $f: \mathbb{R}_{>0} \rightarrow \mathbb{C}$, defined as follows. Associated to a test function is its Mellin transform

$$
\hat{f}(s):=\int_{0}^{\infty} f(x) x^{s} \frac{d x}{x} .
$$

The vector space $\mathcal{A}_{\delta}$ consists of those functions $f(x)$ whose Mellin transform is analytic in the strip $\frac{1}{2}-\delta<\Re(s)<\frac{1}{2}+\delta$, and extends continuously to the boundary of the strip, We put a metric on test functions given by the uniform norm on the closed strip, $d\left(f_{1}, f_{2}\right):=$ $\sup _{s \in S_{\delta}}|f(s)-g(s)|$. With this topology $\mathcal{A}_{\delta}$ is a complete metric space. We define the involution

$$
\tilde{f}(x):=\frac{1}{x} f\left(\frac{1}{x}\right)
$$

whose effect on Mellin transforms is:

$$
\hat{\tilde{f}}(s)=\hat{f}(1-s) .
$$

We define the space $\hat{\mathcal{A}}_{\delta}$ to be the set of Mellin transforms of the elements of $\mathcal{A}_{\delta}$, regarded as analytic functions in the specified strip.

We define the Weil distribution functional

$$
W[f]:=\sum_{\{\rho: \xi(\rho)=0\}}{ }^{\prime} \hat{f}(\rho),
$$

in which' means that the (possibly conditionally convergent) sum is interpreted as $\lim _{T \rightarrow \infty} \sum_{|\rho| \leq T}$. Under suitable conditions $W[f]$ is a continuous linear functional on the allowed set of test functions $\mathcal{A}$. We then define the trace functional

$$
T[f]:=\hat{f}(0)+\hat{f}(1)-W[f]
$$

The quantity $T[f]$ is sometimes called the "spectral side" of the "explicit formula" of prime number theory.

The "explicit formula" in trace form is an formula for $T[f]$ for a suitable set of test functions $f$, taking the shape

$$
T[f]=\sum_{\nu} W_{\nu}(f)
$$

in which $W_{\nu}(f)$ is a contribution associated to each (non-archimedean or archimedean) place $\nu$ of the given field $K$. The right hand side of (9.5) is sometimes called the "arithmeticgeometric" side of the explicit formula. We shall not be concerned with the exact form of the arithmetic-geometric side here, the individual terms $W_{\nu}(f)$ of which can be expressed in various interesting ways, see Burnol [5], Haran 24]. For general automorphic representations $\pi$ the terms $W_{\nu}(f)$ represent contributions from individual terms in the Euler product factorization of $\xi(s, \pi)$. A version of the "explicit formula" in the trace formulation is given in Patterson 
[42, Sect. 3.6]. In order to get an unconditional result one must use a test function space $\mathcal{A}_{\delta}$ with $\delta>\frac{1}{2}$. A version of the "explicit formula" for principal automorphic $L$-functions over $G L(n)$ is given in Rudick and Sarnak [4, Prop. 2.1].

One hope is that the "explicit formula" (9.5) can be interpreted as a Lefschetz trace formula coming from a dynamical system acting on an (unknown) "geometric" object, in which $T[f]$ is to be viewed as a distributional trace with test functions $f$ living on cohomology groups of the geometric object, and the arithmetic side giving data arising from fixed points or periodic orbits on the geometric object. This viewpoint is taken in Deninger [14, 16] [17, 18].

Weil originally formulated the explicit formula in the covariance form given by

$$
W[f]=-\sum_{\nu} W_{\nu}(f)+W_{0}(f)+W_{1}(f) .
$$

This is a rearrangement of the terms in the "trace form" equality, in which we define

$$
W_{0}(f):=\hat{f}(0)=\int_{0}^{\infty} \frac{1}{x} f(x) \frac{d x}{x},
$$

and

$$
W_{1}(f):=\hat{f}(1)=\int_{0}^{\infty} f(x) \frac{d x}{x} .
$$

Attached to this quantity Weil introduced the scalar product

$$
\langle f, g\rangle_{\mathcal{W}}:=W[f * \tilde{\bar{g}}]=\sum_{\rho} \hat{f}(\rho) \overline{\hat{g}(1-\bar{\rho})} .
$$

Weil's criterion for the Riemann hypothesis is that this scalar product be positive semidefinite on a suitable space of test functions, which could be taken to be $\mathcal{A}_{\delta}$ for any $\delta>\frac{1}{2}$, for example. This scalar product will be indefinite if the Riemann hypothesis does not hold. We may transport this scalar product forward to the Mellin-transformed space $\hat{\mathcal{A}}$ as

$$
\langle\hat{f}, \hat{g}\rangle_{\mathcal{W}}:=\langle f, g\rangle_{\mathcal{W}}
$$

The Weil functional $W[f]$ has a natural interpretation in the function field case, see Haran [25].

One feature of the covariance form (9.6) of the "explicit formula" compared to the trace form is that one can make sense of the functional $W[f]$ on a larger set of test functions than those allowed in the trace form, permitting test functions that have singularities at $s=0$ and $s=1$. However the right hand side of (9.6) must then be redefined as a limit as $T \rightarrow \infty$, with each local term computed using cutoffs at $x=\frac{1}{T}$ near zero and at $x=T$ near $\infty$. This was done in the computations in [4. This is a "regularization" of the right side of (9.6) because the two terms $W_{0}(f)$ and $W_{1}(f)$ diverge as the cutoff parameter $T \rightarrow \infty$. Note that these two terms are present only for the trivial representation $\pi_{\text {triv }}$ on GL(1), and are absent from the analogous formula for all other cuspidal automorphic representations $\pi$. In these other cases the analoguous formulas have $T[f]=-W[f]$, so the "trace form" and "covariance form" essentially coincide.

The vector space $\mathcal{L}$ of $\mathrm{Li}$ test functions makes sense for this extended covariance form of the "explicit formula" with a cutoff parameter, and not for the trace form. As noted in $\S 3$, it consists exclusively of rational functions which have poles either at $s=0$ or $s=1$, or both. In consequence the trace function $T[f]$ is undefined for every Li test function. For example, if $g_{n}(x)$ denotes the test function on $\mathbb{R}_{>0}$ whose Mellin transform

$$
G_{n}(s) \hat{g}_{n}(s)=\int_{0}^{\infty} g_{n}(x) x^{s-1} d x
$$


gave the Li test functions $G_{n}(s)$ in (3.2) then $\hat{g_{n}}(0)$ is infinite, for each $n \geq 1$. In [4, Lemma $2]$ the functions $g_{n}(x)$ were explicitly determined, as

$$
g_{n}(s)=\left\{\begin{array}{cl}
P_{n}(\log x) & \text { if } 0<x<1 \\
\frac{n}{2} & \text { if } x=1 \\
0 & \text { if } x>1 .
\end{array}\right.
$$

where $P_{n}(x)$ is the polynomial

$$
P_{n}(x)=\sum_{j=1}^{n}\left(\begin{array}{l}
n \\
j
\end{array}\right) \frac{x^{j-1}}{(j-1) !}
$$

Nevertheless the Weil scalar product of $g_{n}$ with other functions remains well-defined in the covariance form of the "explicit formula" when a cutoff version is used. In terms of the cutoff version of the definition, the unbounded contribution from $\hat{g_{n}}(0)$ above as $T \rightarrow \infty$ is offset by a corresponding divergence coming from the finite primes in the "explicit formula." The Weil distribution functional $W[f]$ is well-defined for all $f \in \mathcal{L}$, as is the Weil scalar product, whether or not the Riemann hypothesis holds. 


\section{References}

[1] E. W. Barnes, On the expression of Euler's constant as a definite integral, Messenger of Math. 33 (1903), 59-61.

[2] P. Biane, J. Pitman and M. Yor, Probability laws related to the Jacobi theta and Riemann zeta functions, and Brownian excursions, Bull. Amer. Math. Soc. 38 (2001), 435-465.

[3] E. Bombieri, Remarks on Weil's quadratic functional in the theory of prime numbers I, Rend. Mat. Acc. Lincei, Ser. IX, 11 (2000), 183-233.

[4] E. Bombieri and J. C. Lagarias, Complements to Li's criterion for the Riemann hypothesis, J. Number Theory 77 (1999), 274-287.

[5] J.-F. Burnol, The explicit formula in simple terms, eprint: arxiv math.NT/9810169 v2 22 Nov 1998.

[6] J.-F. Burnol, Sur les Formules Explicites I: analyse invariante, C. R. Acad. Sci. Paris, Serie I, 331 (2000), 423-428.

[7] P. Cartier and A. Voros, Un nouvelle interprétation de la formule des traces de Selberg, in: The Grothendieck Festscrift, Vol. II, Birkháuser: Boston 1990, pp. 1-67.

[8] M. Coffey, Relations and positivity results for the derivatives of the Riemann $\xi$-function, J. Comput. Appl. Math. 166 (2004), 525-534.

[9] M. Coffey, paper in preparation.

[10] J. Cogdell, Analytic theory of $L$-functions for $G L_{n}$, in: J. Bernstein and S. Gelbart, Eds., An Introduction to the Langlands Program, Birkhäuser: Boston 2003, pp. 197-228.

[11] H. Cramér, Studien über die Nullstellen der Riemannschen Zetafunktion, Math. Zeitschr. 4 (1919), 104-130.

[12] H. Davenport, Multiplicative Number Theory. Third Edition. Revised and with a preface by H. L. Montgomery, Springer-Verlag: New York 2000.

[13] C. Deninger, Local $L$-factors of motives and regularized determinants, Invent. Math. 107 (1992), 135-150.

[14] C. Deninger, Lefschetz trace formulas and explicit formulas in analytic number theory, J. Reine Angew. 441 (1993), 1-15.

[15] C. Deninger, Motivic L-functions and regularized determinants, in: Motives, Proc. Symp. Pure Math., vol. 55, part I, Amer. Math. Soc.: Providence, 1994, 707-743.

[16] C. Deninger, Evidence for a cohomological approach to analytic number theory, in: First European Congress of Mathematics, Vol I (Paris 1992), Birkhäuser: Basel 1994, pp. 491510.

[17] C. Deninger, Some analogies between number theory and dynamical systems on foliated spaces, Proc. Int. Cong. Math. Vol. I (Berlin 1998), Doc. Math. 1998, Extra Vol. 1, pp. $163-186$. 
[18] C. Deninger, On the nature of the "explicit formulas" in analytic number theory- A simple example, in: Number Theoretic Methods (Iizuka, 2001), Dev. Math. Vol. 8, Kluwer: Dordrecht 2002, pp. 97-118.

[19] C. Deninger and M. Schröter, A distribution-theoretic proof of Guinand's functional equation for Cramér's V-function, J. Lond. Math. Soc. 52 (1995), 48-60.

[20] I. M. Gelfand and D. Kazhdan, Representation of the group $G L(n, K)$ where $K$ is a local field, pp. 95-118 in: Lie Groups and Their Representations, John Wiley \& Sons: New York 1974.

[21] S. Gelbart and S. D. Miller, Riemann's zeta function and beyond, Bull. Amer. Math. Soc. 41 (2004), 59-112. [eprint: arXiv math.NT/0309478

[22] R. Godemont and H. Jacquet, Zeta fuctions of simple algebras, Lecture Notes in Math., Vol. 260, Springer-Verlag: Berlin 1972.

[23] A. P. Guinand, Fourier reciprocities and the Riemann zeta-function, Proc. London Math. Soc. 51 (1949) 401-414.

[24] S. Haran, Riesz potentials and explicit sums in arithmetic, Invent. Math. 101 (1990), 697-703.

[25] S. Haran, Index theory, potential theory and the Riemann hypothesis, in: L-Functions and Arithmetic (Durham 1989), Cambridge Univ. Press 1991, pp. 257-270.

[26] S. Haran, The Mysteries of the Real Prime, Oxford Univ. Press: Oxford 2001.

[27] G. Ilies, Cramér functions and Guinand equations, Acta Arith. 105 (2002), 103-118.

[28] H. Iwaniec and E. Kowalski, Analytic Number Theory, Amer. Math. Soc.: Providence, RI 2004.

[29] H. Iwaniec and P. Sarnak, Perspectives on the analytic theory of $L$-functions, GAFA 2000 (Tel Aviv 1999), Geom. Funct. Anal. 2000, Special Volume, Part II, 705-741.

[30] H. Jacquet, Principal $L$-functions of the linear group, pp. 63-86 in: Automorphic Forems, Representations and L-Functions, Proc. Symp. Pure Math. 33, Part 2, Amer. Math. Soc.: Providence, RI 1979.

[31] H. Jacquet and J. A. Shalika, On Euler products and the classification of automorphic representations I, Amer. J. Math. 103 (1981), 499-558.

[32] H. Jacquet and J. A. Shalika, On Euler products and the classification of automorphic representations II, Amer. J. Math. 103 (1981), 777-815.

[33] J. Jorgenson and S. Lang, Guinand's theorem and functional equations for the Cramér functions, J. Number Theory 86 (2001), 351-367.

[34] X.-J. Li, The positivity of a sequence of numbers and the Riemann hypothesis, J. Number Theory 65 (1997), 325-333.

[35] X.-J. Li, Explicit formulas for Dirichlet and Hecke L-functions, Illinois J. Math, to appear. 
[36] X.-J. Li, An explicit formula for the Euler product of Hecke polynomials, eprint: arXiv math.NT/0403148 9 Mar 2004.

[37] X.-J. Li, An explicit formula for Hecke $L$-functions, eprint: arXiv math.NT/0403119 6 Mar 2004.

[38] W.-Z. Luo, Z. Rudnick and P. Sarnak, On Selberg's eigenvalue conjecture, Geom. Funct. Anal. 5 (1995), 387-401.

[39] W.-Z. Luo, Z. Rudnick and P. Sarnak, On the generalized Ramanujan conjecture for GL(n). pp. 301-310 in: Automorphic forms, automorphic repesentations and arithmetic (Fort Worth, TX 1996), Proc. Symp. Pure Math. Vol 66, Part 2, Amer. Math. Soc.: Providence, RI 1999.

[40] K. Maslanka, An effective method of computing Li's coefficients and their unexpected properties, eprint:arXiv: math.NT/0402168 v2.

[41] C. Moeglin and J.-L. Waldspurger, Pôles des fonctions $L$ de paires pour $G L(N)$, app. to Le spectre résiduel de $G L(N)$, Ann. Sci. Ecole Norm. Sup. (4-ème série) 22 (1989), 605-674.

[42] S. J. Patterson, An introduction to the theory of the Riemann zeta function, Cambridge U. Press: Cambridge 1988.

[43] R. Remmert Classical Topics in Complex Function Theory, Springer-Verlag: New York 1998. (Translation of German edition 1991).

[44] Z. Rudnick and P. Sarnak, Zeros of principal $L$-functions and random matrix theory, Duke Math. J. 81 (1996), 269-322.

[45] F. Shahidi, On certain L functions, Amer. J. Math. 103 (1981), 297-355.

[46] E. C. Titchmarsh, The Theory of Functions, Second Ed., Oxford U. Press 1944, reprint 1967.

[47] A. Voros, Spectral functions, special functions and the Selberg zeta function, Comm. Math. Phys. 110, 439-465.

[48] A. Voros, Spectral zeta functions, in: Zeta Functions in Geometry, Adv. Studies in Pruer Math., Vol 24, 1992, pp. 327-358.

[49] A. Voros, Zeta functions for the Riemann zeros, Ann. Inst. Fourier 53 (2003), 665-699.

[50] A. Voros, A sharpening of Li's criterion for the Riemann hypothesis, eprint: arXiv math.NT/0404213.

[51] A. Weil, Sur les "formules explicites" de la théories des nombres premiers, Meddelanden Från Lunds Univ. Mat. Sem. (dedié a M. Riesz) (1952), 252-265. (Also: Euvres Scientifiques - Collected Papers, Springer-Verlag, Corrected Second Printing 1980, Vol.II, 48-61.) 